\title{
Important Properties of Clay Content of Lateritic Soils for Engineering Project
}

\author{
N. O. Adebisi ${ }^{1}$, G. O. Adeyemi ${ }^{2}$, O. S. Oluwafemi ${ }^{3}$ \& S. P. Songca ${ }^{4}$ \\ ${ }^{1}$ Department of Earth Sciences, Faculty of Science, Olabisi Onabanjo University, Ago-Iwoye, Nigeria \\ ${ }^{2}$ Department of Geology, Faculty of Science, University of Ibadan, Ibadan, Nigeria \\ ${ }^{3}$ Department of Chemistry and Chemical Technology, Walter Sisulu University, Mthatha Campus, Mthatha, \\ South Africa \\ ${ }^{4}$ Executive Dean, Walter Sisulu University, Tecoma, East London, South Africa \\ Correspondence: Songca S. P., Executive Dean, Walter Sisulu University, PO Box 19712, Tecoma, East London, \\ South Africa. E-mail: spsongca@wsu.ac.za
}

Received: September 4, 2012 Accepted: October 30, 2012 Online Published: April 25, 2013

doi:10.5539/jgg.v5n2p99

URL: http://dx.doi.org/10.5539/jgg.v5n2p99

\begin{abstract}
Clay-sized particles have been shown to control the engineering performance of lateritic soils, while the mode of formation and mineralogical composition of parent rocks in evaluating properties peculiar to clay-sized particles are yet to be a subject of serious research. Fresh Gneiss (GN), Quartz-schist (QS) and Granite (GR) were sampled in parts of Southwestern Nigeria. Thirty samples each of disturbed and undisturbed soils were also obtained at depths of 1.0, 1.5, 2.0, 2.5 and $3.0 \mathrm{~m}$ from profiles over GN, QS and GR. X-ray fluorescence and $\mathrm{X}$-ray diffraction were employed to determine the major oxide geochemistry and clay mineralogy respectively, while grain-size distribution, plasticity characteristics, undrained cohesion $(\mathrm{Cu})$ and volume compressibility $(\mathrm{Mv})$ were determined following the British Standards (BS-1337).

Parent rocks petrography reveals quartz and muscovite in QS, and quartz, alkali feldspars and biotite in GN and GR. $\mathrm{SiO}_{2} / \mathrm{Fe}_{2} \mathrm{O}_{3}+\mathrm{Al}_{2} \mathrm{O}_{3}$ indicates that soils form GN and GR fall into a class different from QS. Kaolinite (52.3-75.5\%) formed the dominant clay mineral in the soils with subordinate amount of illite (2.3-17.6\%), while 1.9 and $0.9 \%$ of smectite occurred at $3.0 \mathrm{~m}$ depth in soils over GN and GR respectively. The relationship between $\mathrm{I}_{\mathrm{p}}$ and $\mathrm{Fe}_{2} \mathrm{O}_{3}$ taking cognizance of parent rock factors reveals that the form of iron oxide that reduces the plasticity of lateritic soils. The mode of formation and mineralogical composition of parent rocks caused variation in cohesion and compressibility characteristics of lateritic soils.
\end{abstract}

Keywords: clay, lateritic, rock, mineralogy and properties, engineering performance

\section{Introduction}

\subsection{Importance of Lateritic Soils for Civil Engineering Projects}

In most parts of the tropical areas of the world, lateritic soils are extensively encountered. Clay is an important component of lateritic soils, especially when assessed for civil engineering purposes (Townsend, 1985). In this case the engineering geologist is expected to give predictions of the behavior of the soils in projects involving preliminary designs. The field of clay properties is diverse and complex; therefore, a study on clay properties in geotechnical engineering warrants a descriptive review in some detail. It is the aim of this paper to discuss selective properties in order to provide a workable compendium.

\subsection{Compositional Features}

In this paper, results of laboratory tests on the clay content of lateritic soils derived from three different parent rocks are presented. Particular emphasis is given to compositional features and mode of geological formation of the parent rocks. Furthermore, elemental abundances of the soils in terms of major oxides are employed to explain the degree of laterisation. Clay mineralogical composition is also discussed with reference to kaolinite, illite and montmorillonite, which are applicable to engineering practice. Besides, minerals present in the soils are also inferred from the consistency limits in general. 


\subsection{Plasticity Characteristics}

Plasticity characteristics, mainly cohesive strength and derived limits in addition to compressibility of the clay are analyzed. The coefficient of volume compressibility is related to the amount of settlement, and has been employed to explain the amount of settlement a foundation soil would experience on application of building load. It is suggested that these geological fundamentals would be of practical assistance to civil engineers faced with challenges of abnormal soil behavior due to clay content in the construction industry.

\subsection{Parent Rocks and Soil Formation of the Study Area}

The Precambrian rocks of the Basement Complex in Southwestern Nigeria include gneiss, quartzite, quartz-schist and granite (Jones \& Hockey, 1964). Unlike granite which is formed under igneous activity, gneiss and quartz-schist are metamorphic rocks and are formed under similar geological conditions. Faniran and Jeje (1983), Wilson (1986), Adeyemi (1992), Adebisi (1999) and Adebisi (2003) explain the varying degrees of weathering processes experienced by the parent rocks through the action of water and other agents to form laterized profiles of soils with various thicknesses as influenced by topography and vegetation with strong influence of the tropical climate. The tropical climate is characterized by alternating dry and wet seasons (Oke, 1974), and it is an important condition in the formation of the lateritic soil.

\subsection{Grain-Size Distribution}

Textural analysis of lateritic soils by particle size provides a useful engineering classification system from which a considerable amount of empirical data can be obtained. It involves the mechanical sieving for coarse particles, and the hydrometer analysis for fine fractions, such as clay. Grading curves are useful to show grain-size distribution. Soil consists of an assembly of discrete soil particles of various sizes. The object of a grain size analysis is to group these particles into separate ranges of sizes and to determine the relative proportion by weight of each size range. The hydrometer analysis method employs sieving and sedimentation of a soil/water/dispersant suspension to separate the particles. The sedimentation technique is based on an application of Stokes' law to a soil/water suspension and periodic measurement of the density of the suspension.

\subsection{Consistency Limits}

Consistency limits provide a measure of the four states: solid, semi-solid, plastic and liquid, that are possible for a soil depending on water content. Because the behavior of a soil and therefore its engineering properties is different in each of these four states, the boundary between each state can be defined based on a change in the soil's behavior. Consistency limits are used to distinguish between silt and clay, and they can be used to distinguish between different types of silts and clays. Albert Atterberg was the first to develop procedures for describing the limit consistency of fine-grained soils on the basis of water content (Das, 1983). These were were later refined by Arthur Casagrande (1932). The consistency limits of a soil are used extensively to discriminate between different kinds of soils within a broad category from silt to clay-sized particles. The plasticity index $\left(\mathrm{I}_{\mathrm{P}}\right)$ versus liquid limit $\left(\mathrm{L}_{\mathrm{L}}\right)$ plots on the chart of Casagrande are widely used for classification of soils.

\subsection{Major Oxides Geochemistry}

Abundances of major elements of a lateritic soil and those of their respective pulverized parent rocks, are obtainable through X-Ray fluorescence (X-RF) spectrometry of its clay content. Other modern methods of conducting these measurements include inductively coupled plasma mass spectroscopy (ICP-MS) and optical spectroscopy (ICP-OS). It is common practice to express major elements as weight percentage of oxides (e.g. $\mathrm{SiO}_{2}$ ) and trace elements in parts per million (ppm). Mixing major elements expressed as weight percentage of oxides with elements reported without oxides expressed in parts per million, is generally avoided as it leads to scale differences.

\subsection{Clay Mineralogy}

Crystalline cay minerals are hydrated silicates with layer or chain lattices consisting of sheets of silica tetrahedra arranged in hexagonal form condensed with octahedral layers; they are usually of small particle size. Identification, characterization and understanding of properties of different clay minerals can help in evaluation of lateritic soils for classification and engineering performance. X-Ray diffraction (X-RD) method is suitable for identifying and quantifying the clay minerals present in a lateritic soil. Clay minerals are produced mainly from the chemical weathering and decomposition of feldspars, such as orthoclase and plagioclase and some mica. The mineralogical composition of a parent rock is a pointer to the composition of soils developed over it (Aleva 1994). Physical weathering produces very coarse soils and gravels consisting of broken particles, but sand and silt mainly consist of mineral grains. Chemical weathering follows under wet and warm conditions of degradation by decomposition and or alteration. Rock-forming minerals such as quartz, hematite and muscovite 
are resistant to chemical weathering, and remain unchanged (Philpotts, 1989; Adeyemi, 1992; Elueze et al., 2004; Ige et al., 2005). Quartz is an enduring mineral found in granite and gneisses.

\subsection{Cohesion and Compressibility}

In geotechnics, cohesion and compressibility are important properties of lateritic soils brought about by clay content. Unlike those properties earlier discussed, which could be determined directly in the laboratory from the clay content of disturbed soils, undrained cohesion is determined in a triaxial test. Volume compressibility of clay is determined in an oedometer consolidation test. Mathematically, the shear strength of a lateritic soil is governed by the Mohr-Coulomb failure criterion.

$$
S=C+P \tan \varnothing
$$

Where $\mathrm{S}$ is the shear stress at failure along any plane and it is the normal stress on that plane. $\mathrm{C}$ and $\varnothing$ are the shear strength parameters; cohesion and angle of shearing resistance. Cohesion is the shear strength parameter due to attractive forces of clay minerals.

Consolidation is a fundamental phenomenon that must be considered to properly understand soil behavior in foundation problems. The measure of the tendency of a soil to decrease in volume under load is called its compressibility $\left(\mathrm{M}_{\mathrm{V}}\right)$. The coefficient of volume compressibility of a lateritic soil can be obtained in an oedometer consolidation test (BS, 1990). For the coefficient of volume compressibility

$$
M v=\frac{d h}{d p}-x \frac{1}{h}
$$

where: $\mathrm{dh}=$ change in thickness, $\mathrm{dp}=$ change in pressure, $\mathrm{h}=$ average of $\mathrm{h} 1 \mathrm{hnd} \mathrm{h} 2$ (thickness of samples). The coefficient of compressibility is the most suitable of all the consolidation parameters for direct estimation of settlement despite its variation with confining pressure.

\section{Methods}

The fieldwork involved geological and pedological mapping of the study area. In-situ geotechnical investigation was conducted by Cone Penetration Testing (CPT) at six locations, two on each horizon of soils developed over gneiss, quartz schist and granite. Fresh samples of parent rocks as well as bulk soil samples were collected. A total number of sixty soil samples, consisting of thirty of each of disturbed and undisturbed soils were obtained at $1.0 \mathrm{~m}, 1.5 \mathrm{~m}, 2.0 \mathrm{~m}, 2.5 \mathrm{~m}$ and $3.0 \mathrm{~m}$ depth, from six trial pits, two from the horizons of soils over gneiss, quartz schist and granite bedrock settings. The samples were labeled, described, prepared and transported to the laboratory for geotechnical investigation, including geochemical and clay mineralogical analyses. The index and engineering properties investigated included Natural moisture content (Nmc), Specific gravity of grains (Gs), Grain-size distribution, Consistency limits, Cohesion $(\mathrm{Cu})$ and angle of internal friction $(\varnothing)$, Coefficient of consolidation $(\mathrm{Cv})$ and Coefficient of volume compressibility $(\mathrm{Mv})$. The procedures adopted for tests are in accordance with the British standard BS-5930 (1981), and ASTM standard D 422-90, (ASTM, 1985) for site investigation, which recommend terminology and the Unified Soil Classification System (USCS) to describe and classify soils for engineering purposes. Other procedures include BS-8004: (1986) and BS-1337 (1990), code of practice for foundations and soil testing respectively. Modifications and special treatments of the studied soils were however, made where necessary.

In order to study the mineralogical composition of the parent rocks thin sections were observed under a petrographic microscope in transmitted light (Figure 2). Textural analysis of lateritic soils by particle size was conducted by mechanical sieving for coarse particles, and by the hydrometer analysis for fine fractions, such as clay. The samples were subdivided and separate analyses were carried out on each sub-sample. The liquid limit $\left(\mathrm{L}_{\mathrm{L}}\right)$ and plastic limit $\left(\mathrm{L}_{\mathrm{p}}\right)$ were determined on air-dried soil samples that passed through the British Standard sieve no. 40 (slot size of $0.425 \mathrm{~mm}$ ) as described by Donker (1974). The plasticity index $\left(\mathrm{I}_{\mathrm{P}}\right)$ versus liquid limit $\left(\mathrm{L}_{\mathrm{L}}\right)$ was plotted on the classification chart of Casagrande.

Abundances of major elements of lateritic soils and that of their respective pulverized parent rocks, were obtainable from X-Ray fluorescence (X-RF) spectrometry of its clay content (Adebisi, 2010). Furthermore, the $\mathrm{X}$-Ray diffraction (X-RD) method was used for identifying and quantifying the clay minerals present in lateritic soils. The amount of settlement of a lateritic soil can be estimated from the measured coefficient of volume compressibility. The coefficient of volume compressibility of the lateritic soils was obtained from the odometer consolidation tests (BS, 1990). Applying the standard of Carter (1983), it becomes possible to express the compressibility characteristics of the soils as shown in Table 8. In order to establish the influence of the parent 
rock factor on the settlements that could be caused by foundation load, the statistical t-test on the coefficient of volume compressibility of the studied soils was carried out and the results are summarized in Table 9.
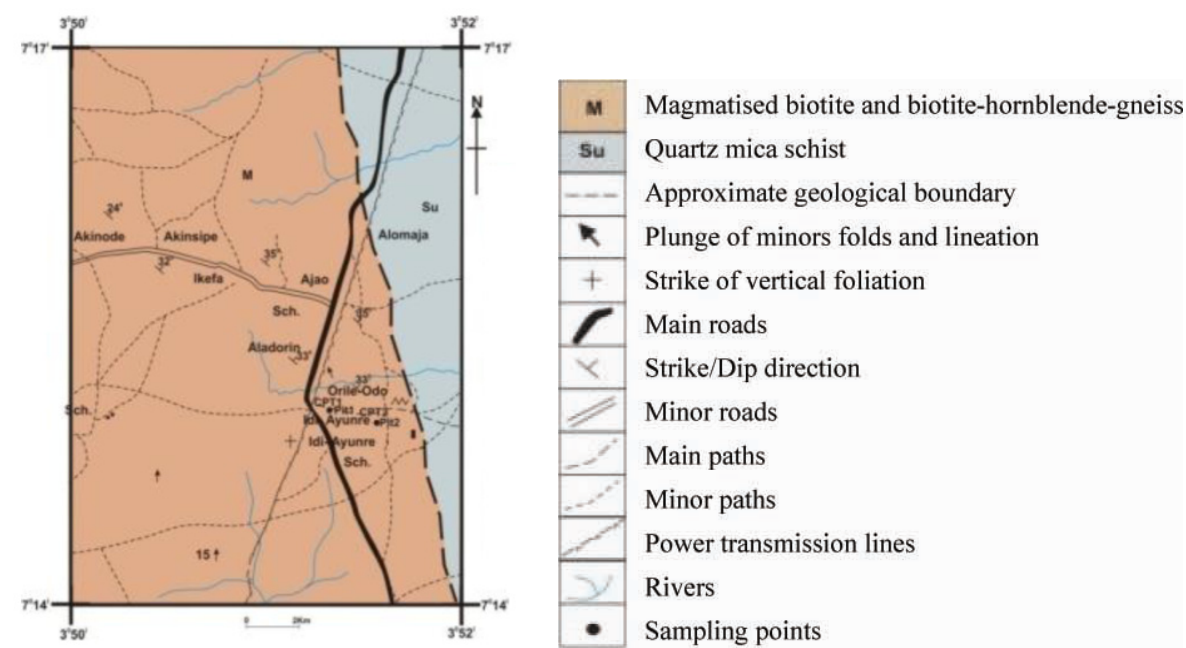

a. Geological map of Idi-Ayunre
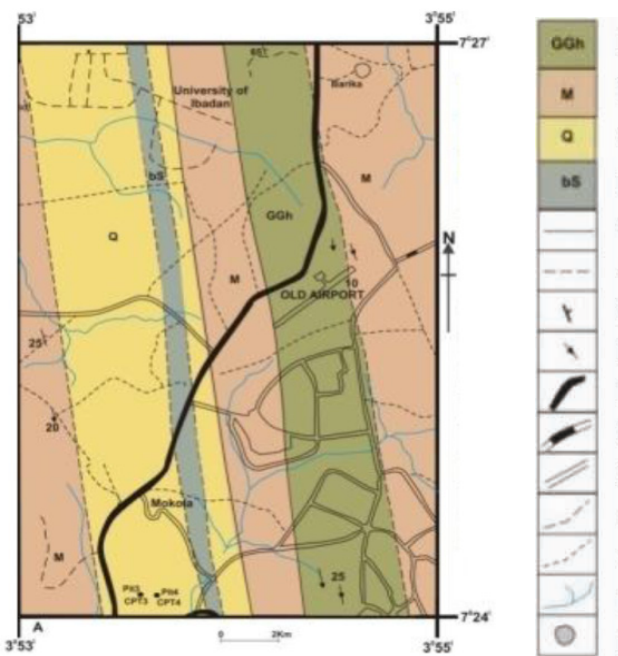

Hornblende-biotite-granite-gneiss

Magmatised biotite and biotite-hornblende-gneiss

Quartzite and Quartz-schist

Biotite gneiss

Geological boundery

Approximate geological boundery

Strike and Dip of foliation

Plunge of minors folds and lineation

Main roads

Secondary roads

Minor roads

Main paths

Minor paths

Rivers

Built up area

b. Geological map of Ibadan
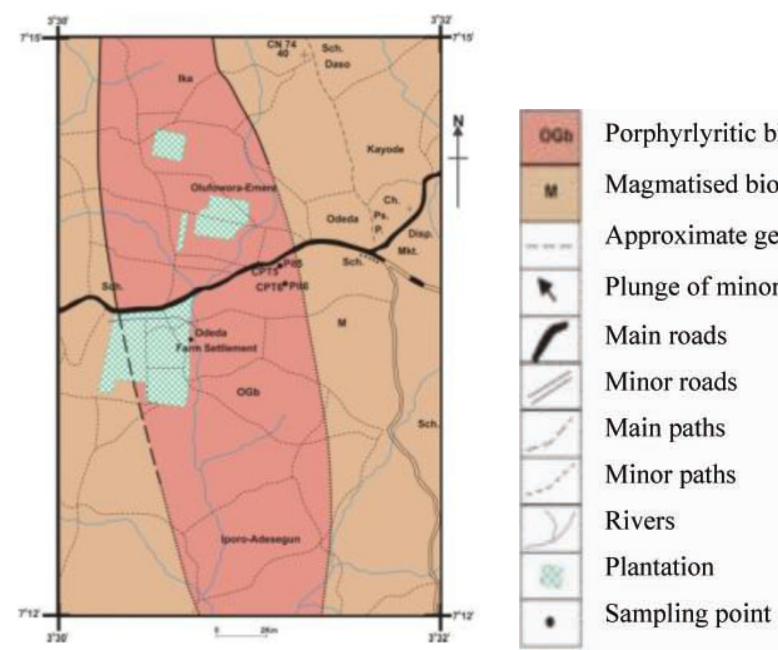

c. Geological map of Odeda

Figure 1. Geological maps form Southwestern Nigeria showing parent rocks (Adebisi, 2010) 


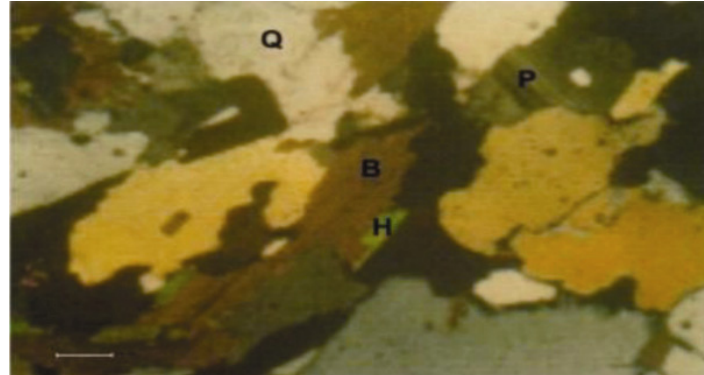

a

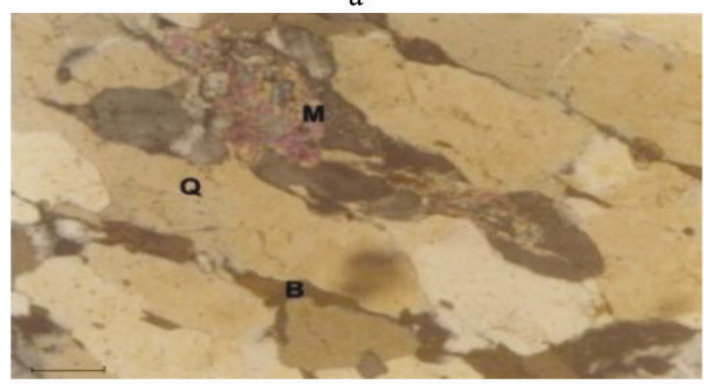

c

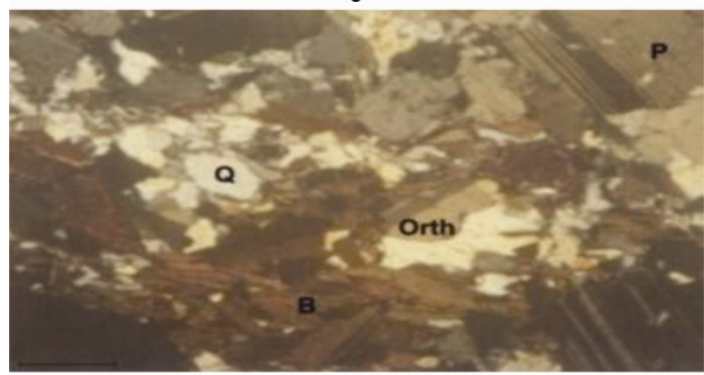

e

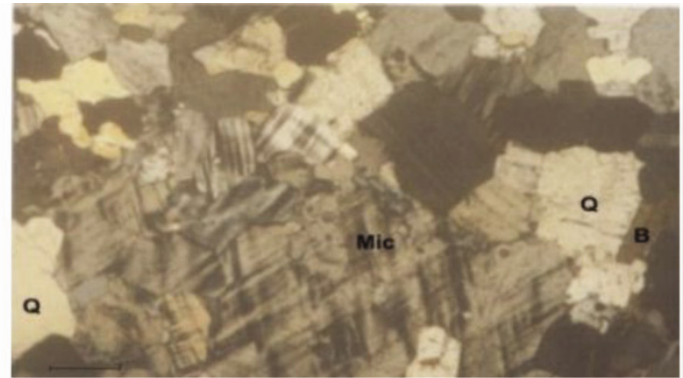

b

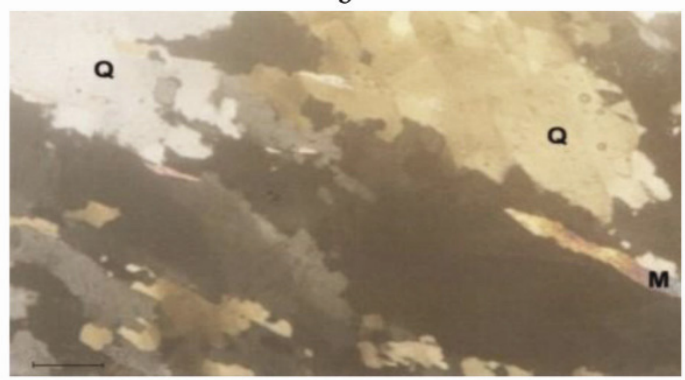

d

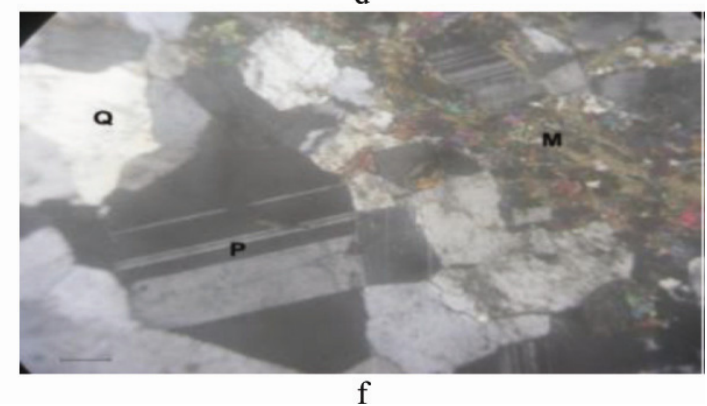

Figure 2. Photomicrographs of gneiss (a \& b), quartz schist (c \& d) and granite (e \& f) in transmitted light; showing muscovite (M), orthoclase (Orth), microcline (Mic), quartz (Q) and biotite (B) (Adebisi 2010). (Bar scale $=2.5 \mathrm{~mm}$ )

\section{Results}

Figure 1 features three geological maps of the sampling areas form Southwestern Nigeria showing parent rocks. It has three geological maps from parts of Southwestern Nigeria showing the various locations of the major rocks which underlie the study areas. These are the parent rocks from which lateritic soils were derived. These soils are widely employed as both construction and foundation materials. Photomicrographs of gneiss, quartz schist and granite in transmitted light, showing muscovite (M), orthoclase (Orth), microcline (Mic), quartz (Q) and biotite (B) are shown in Figure 2. The photomicrographs show thin sections revealing the mineralogical composition of the parent rocks as observed under a petrographic microscope in transmitted light.

The various minerals which make-up the parent rocks are summarized in Table 1. This is possible through a modal analysis of each of the thin sections. This table lists the mineralogical constituents of parent rocks to the lateritic soils. The characteristics of the lateritic profile of the lateritic soils developed over each of the parent rocks are shown in Figure 3. The results of chemical weathering are generally fine grained-soils with separate mineral grains such as clay and clay-silts. Figure 3 illustrates the various profile development of the parent rocks. Grading curves for the soils from the various sampling pits are shown in Figure 4 and these are grain-size distribution curves of combined coarse and fine analyses of the studied soils. Figure 5 shows the Casagrande plasticity chart for classification of the lateritic soils studied. The X-Ray diffraction patterns of the lateritic soils studied are shown in Figure 6. The role of iron oxide found in lateritic soils, in plasticity behavior of the lateritic soil have been stressed by Chandrakaran and Nambiar (1993). Regression analyses between Ip and $\mathrm{Fe}_{2} \mathrm{O}_{3}$ are shown in Figure 7. Figure 7a describes the situation from a purely civil engineering point of view, whereby the parent rock is not taken into consideration. Regression plots of plasticity index against amount of iron oxide were prepared for Quartz-schist-derived soils and Granite derived soils. These are shown in Figure 7. 
Table 1. Mineralogical constituents of parent rocks to the lateritic soils

\begin{tabular}{|c|c|c|c|c|c|c|}
\hline \multirow{3}{*}{ Mineral Constituents (\%) } & \multicolumn{2}{|c|}{ Gneiss } & \multicolumn{2}{|c|}{ Quartz-Schist } & \multicolumn{2}{|c|}{ Granite } \\
\hline & \multicolumn{6}{|c|}{ Sample Number } \\
\hline & 1 & 2 & 3 & 4 & 5 & 6 \\
\hline Quartz & 27 & 40 & 62 & 66 & 34 & 46 \\
\hline Plagioclase & 32 & & - & - & 20 & 34 \\
\hline Orthoclase & - & - & - & - & 36 & - \\
\hline Microcline & - & 39 & - & _ & - & - \\
\hline Biotite & 19 & 5 & 4 & - & 14 & - \\
\hline Hornblende & 10 & - & - & - & - & - \\
\hline Muscovite & - & - & 28 & 20 & - & 18 \\
\hline Others & 12 & 16 & 6 & 14 & 6 & 2 \\
\hline
\end{tabular}
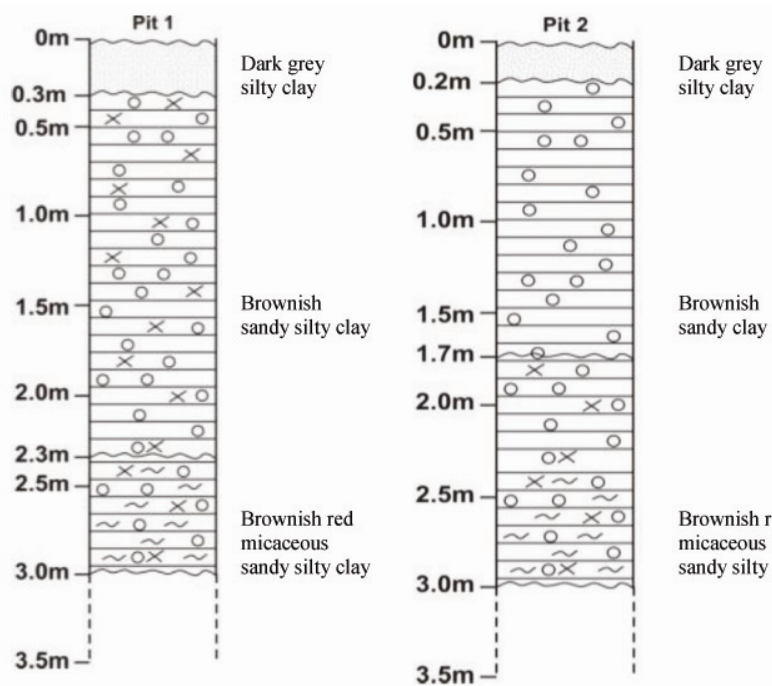

silty clay
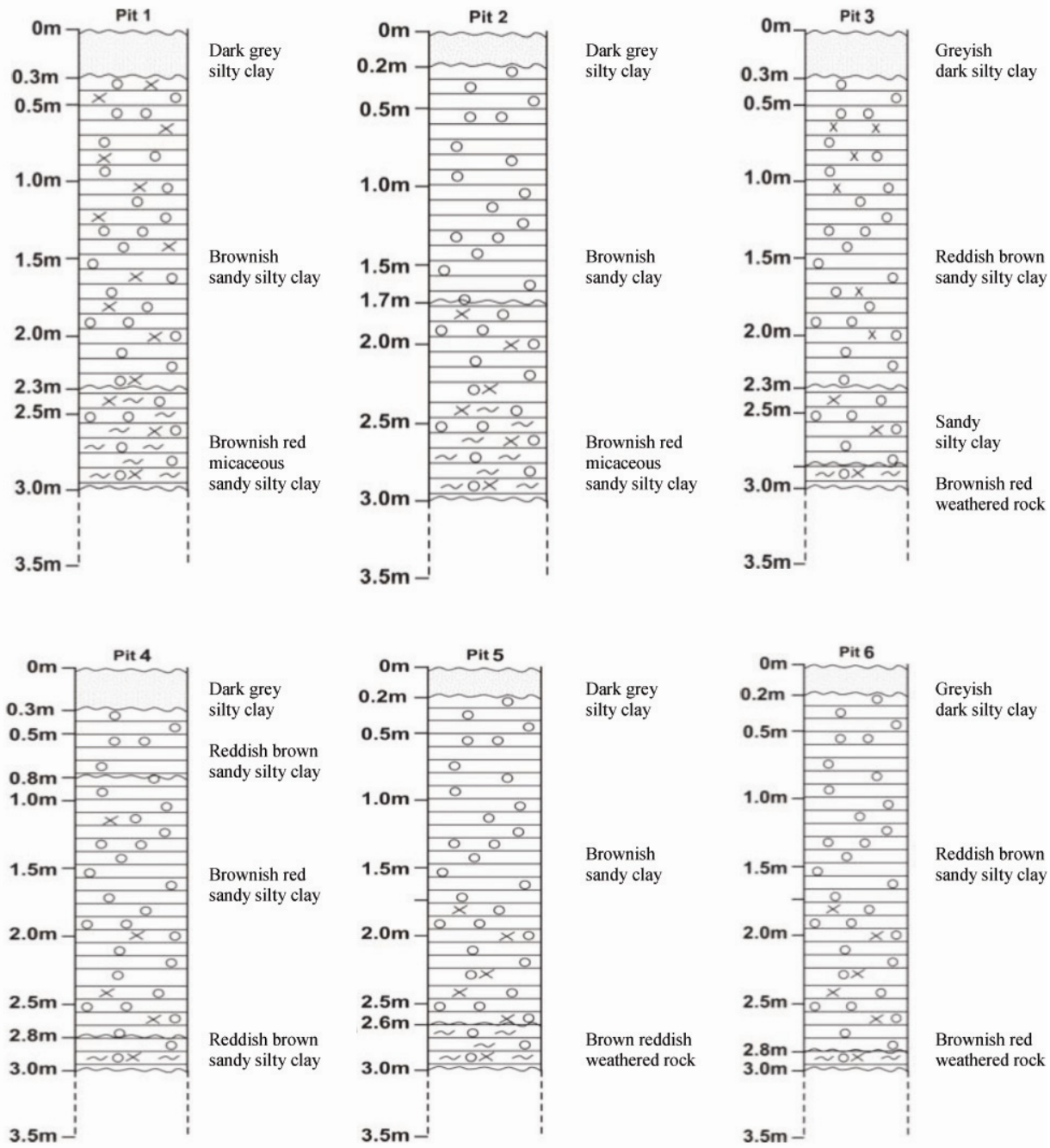

sandy silty

Brownish red sandy silty clay
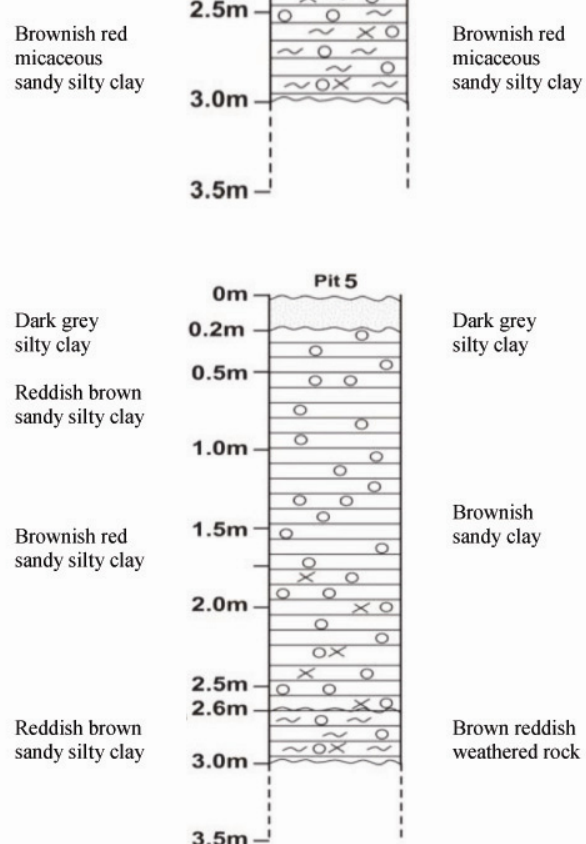

$3.5 \mathrm{~m}$

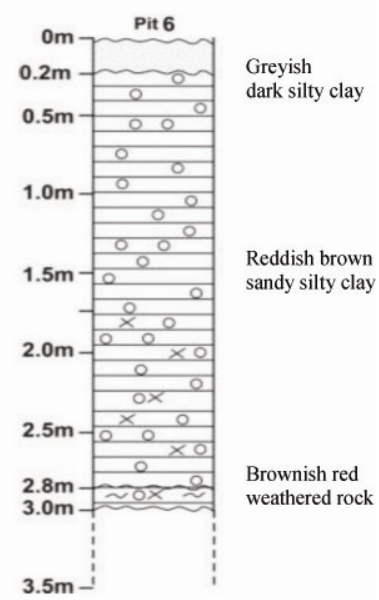

Figure 3. Sketch of lateritic profile characteristics of lateritic soils developed over each of the parent rocks (Adebisi, 2010) 


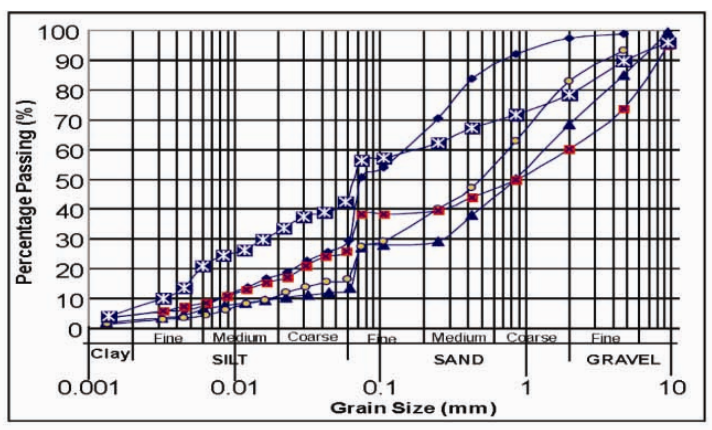

a

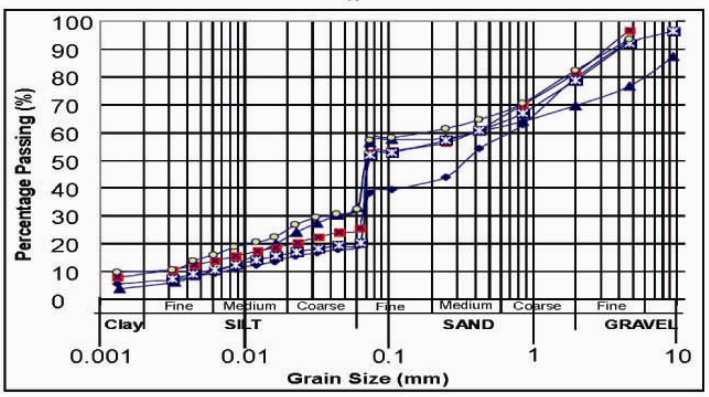

c

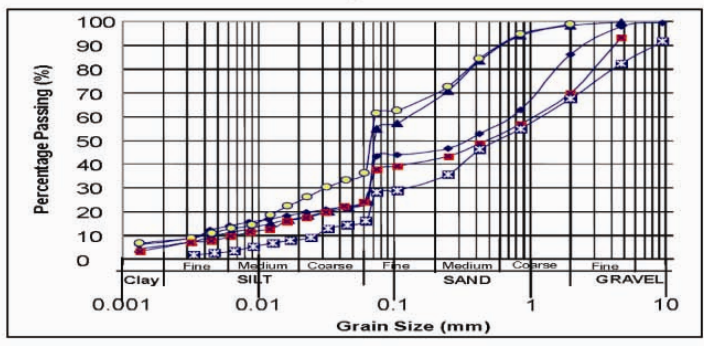

e

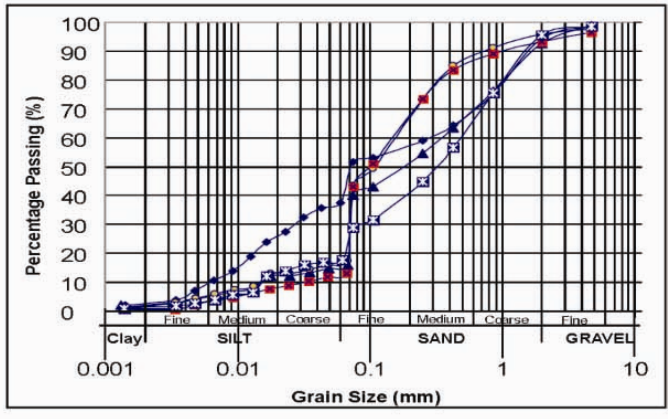

b

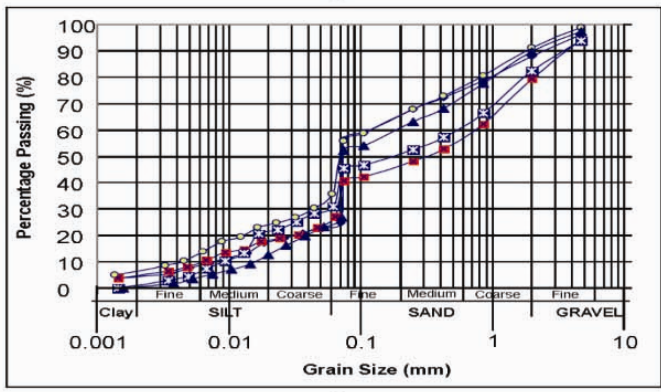

d

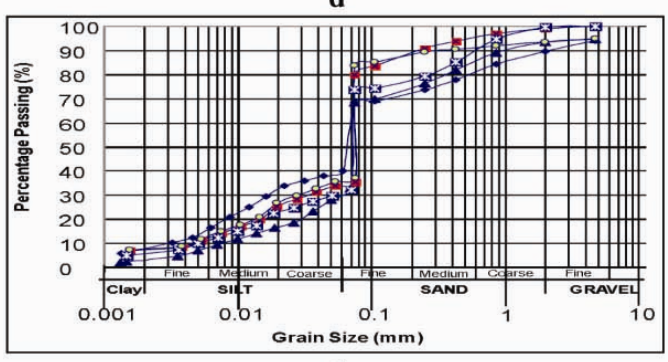

Grain Size $(\mathrm{mm})$

$-1.5 \mathrm{~m}$

$\rightarrow \mathbb{1}-1.0 \mathrm{~m}$

Figure 4. Grading curves a \& b for gneiss-derived soils from Pits 1 and $2, \mathrm{c} \& \mathrm{~d}$ for quartz-schist-derived soils from Pits $3 \& 4$, e \& f for granite-derived soils from Pits $5 \& 6$ (Adebisi, 2010)
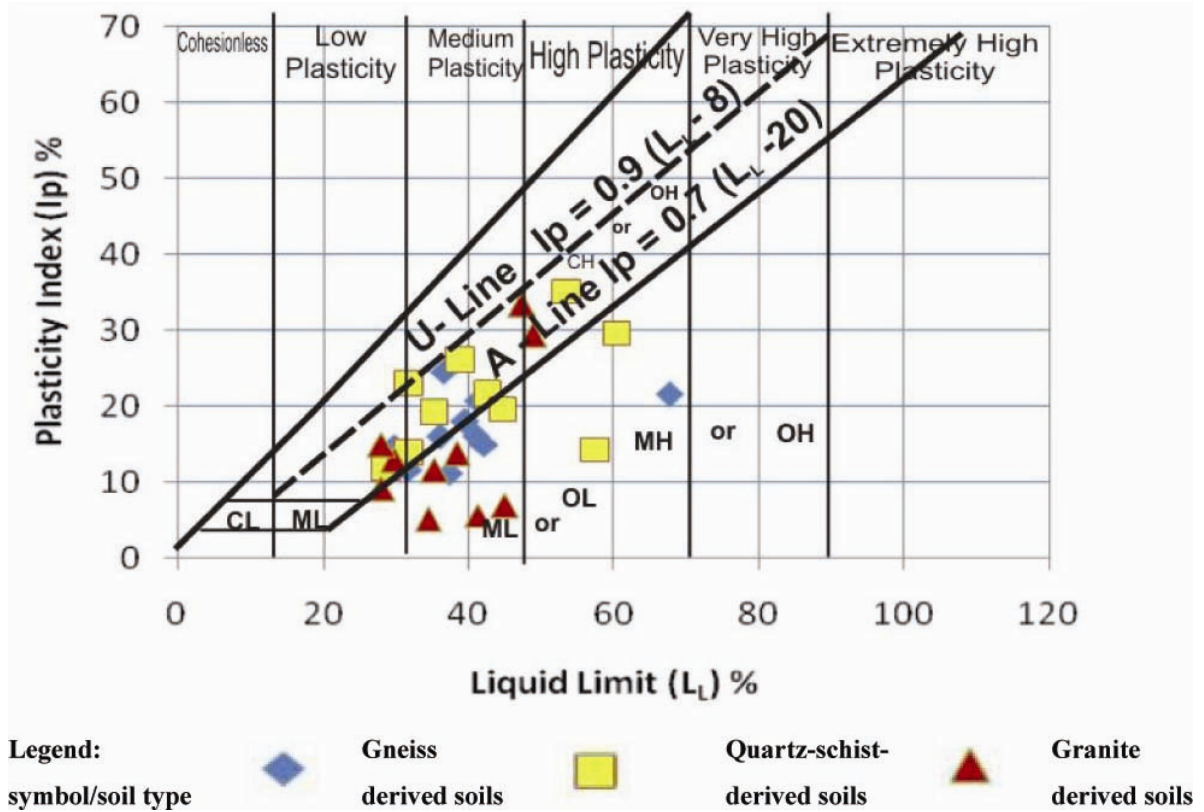

Figure 5. Casagrande's plasticity chart for soil classification 


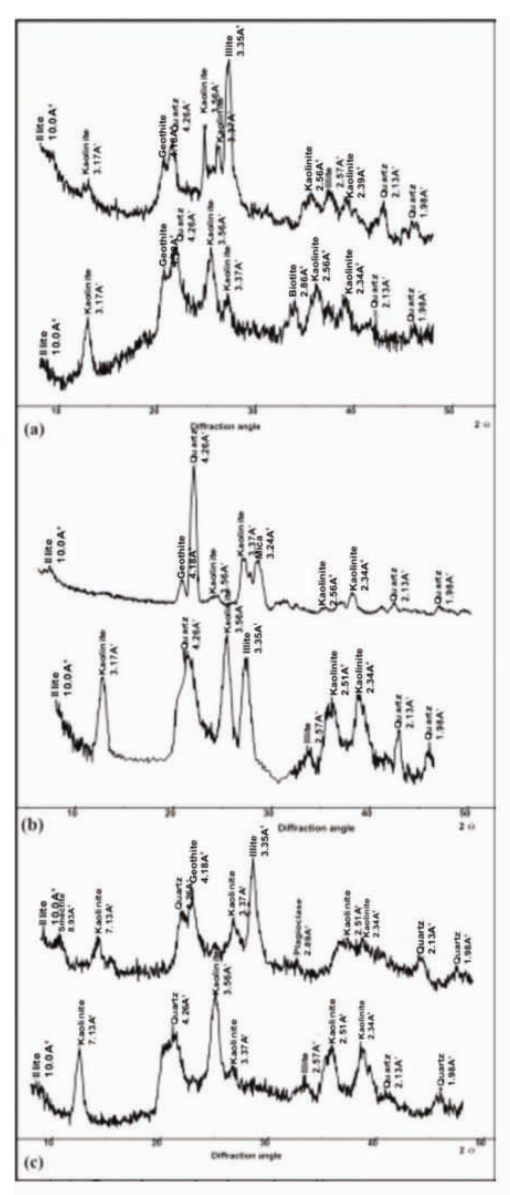

(a) Gneiss-derived soils

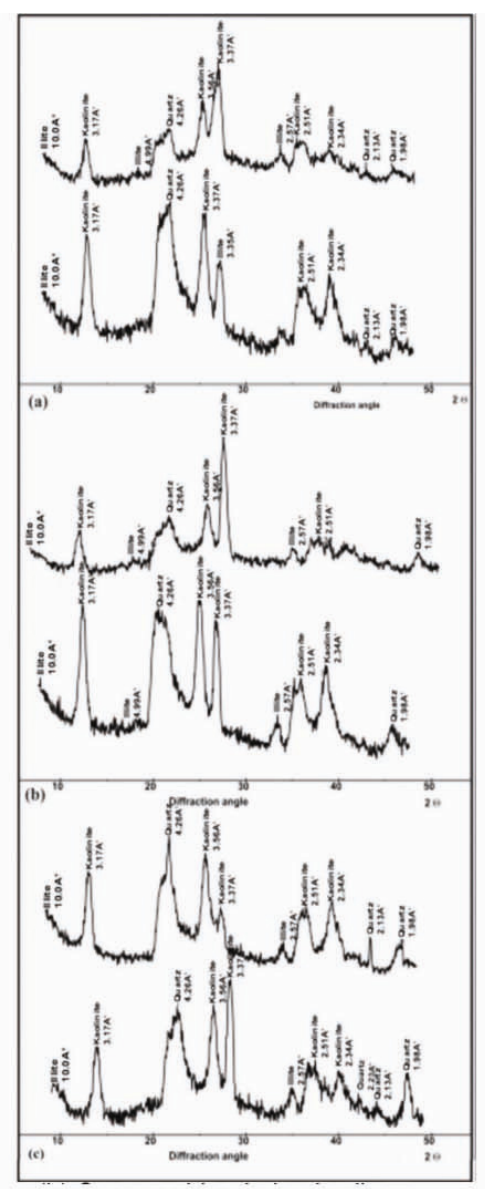

(b) Quartz-schist derived soils

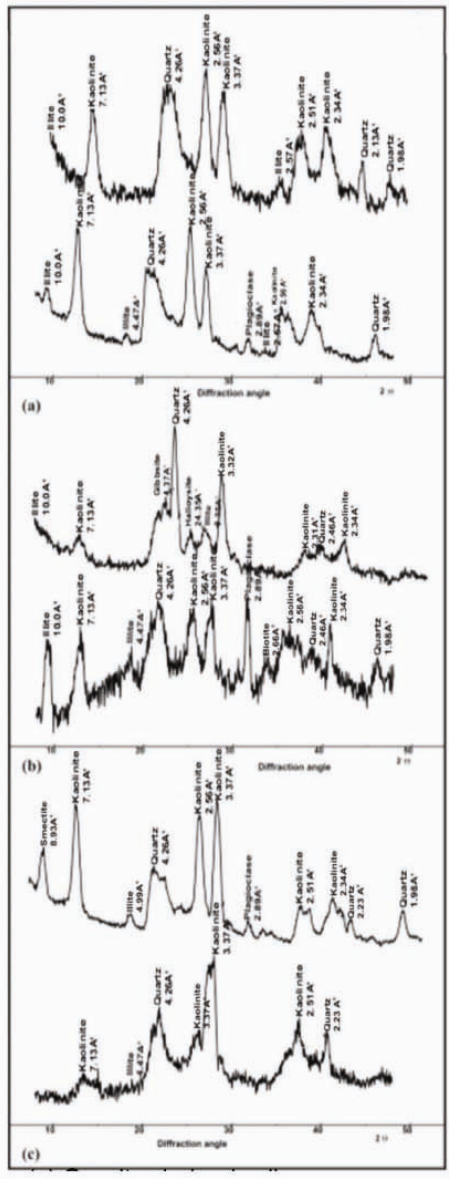

(c) Granite derived soils

Figure 6. X-Ray diffraction patterns of the lateritic soils (Adebis, 2010)
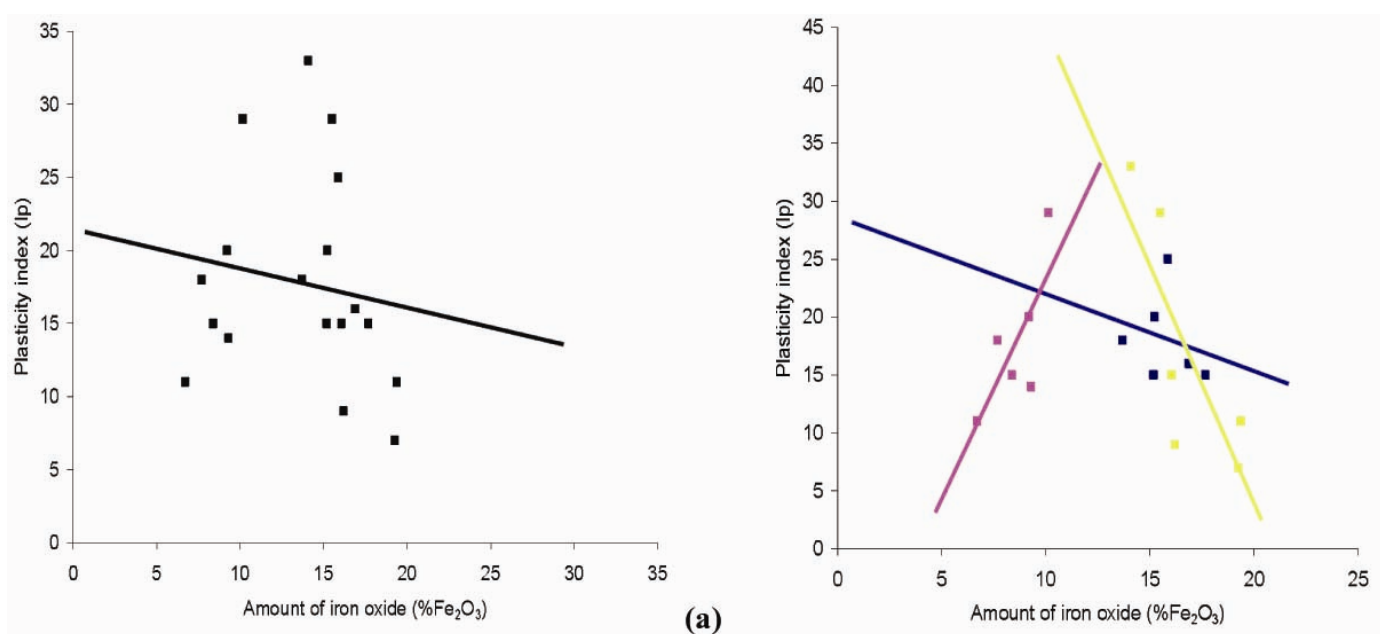

(b)
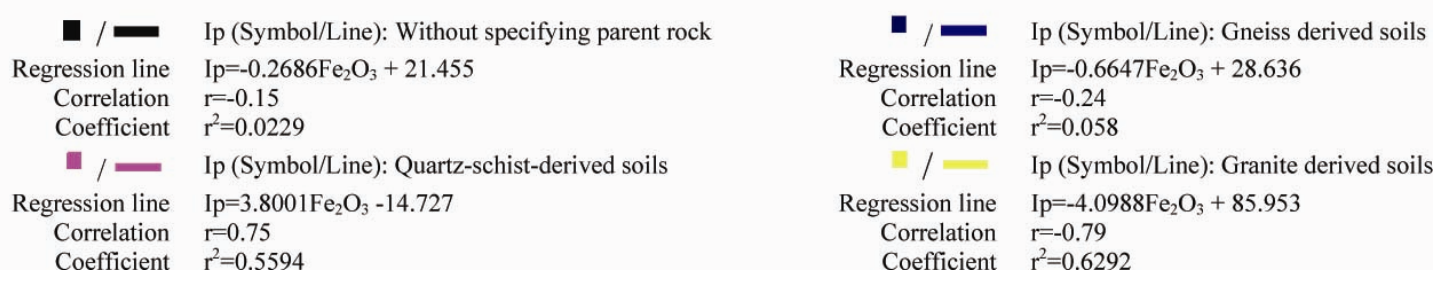

Figure 7. Regression plots of plasticity index against amount of iron oxide (legend below) 
In addition, some of the data are tabulated. Table 1 lists the mineralogical constituents of parent rocks to the lateritic soils. Table 2 contains the grain size distribution of the studied soils. The plasticity characteristics of the studied soils are given in Table 3 . In this paper, both liquid limit $\left(\mathrm{L}_{\mathrm{L}}\right)$ and plastic limit $\left(\mathrm{L}_{\mathrm{p}}\right)$ were determined on air-dried soil samples passing through the British Standard sieve no. 40 (slot size of $0.425 \mathrm{~mm}$ ). Results of the consistency limits test, which describe variation in plasticity of the studied soils are shown in Table 3 . Table 4a contains the consistency index (Ic) of the studied soils and Table $4 \mathrm{~b}$ shows the liquidity index of the studied soils. The major oxides of lateritic soils are shown in Table 5. Table 6 shows clay mineralogical composition of the lateritic soils. Table $7 \mathrm{a}$ gives the consistency limits in relation to clay mineralogy of lateritic soils. Table $7 \mathrm{~b}$ shows the variation in undrained cohesion of lateritic soils with depth.

Table 2. Grain size distribution of the studied soils

\begin{tabular}{|c|c|c|c|c|c|c|}
\hline \multirow[b]{2}{*}{ Soil Type } & \multirow{2}{*}{$\begin{array}{l}\text { Depth } \\
(\mathrm{m})\end{array}$} & \multicolumn{5}{|c|}{ Grain-size Distribution } \\
\hline & & $\begin{array}{c}\text { \% Clay-size } \\
\text { Particles }\end{array}$ & $\begin{array}{c}\% \text { Silt-size } \\
\text { Particles }\end{array}$ & $\%$ Fines & $\begin{array}{c}\text { \% Sand-size } \\
\text { Particles }\end{array}$ & $\begin{array}{c}\text { \% Gravel-size } \\
\text { Particles }\end{array}$ \\
\hline Gneiss-derived Soils & 1.0 & 6.0 & 30.0 & 36 & 59.0 & 5.0 \\
\hline \multirow[t]{4}{*}{ (Trial Pit1) } & 1.5 & 5.0 & 35.0 & 40 & 53.0 & 7.0 \\
\hline & 2.0 & 4.0 & 12.0 & 16 & 78.0 & 5.0 \\
\hline & 2.5 & 3.0 & 8.0 & 11 & 82.0 & 7.0 \\
\hline & 3.0 & 5.0 & 13.0 & 18 & 77.0 & 5.0 \\
\hline Gneiss-derived Soils & 1.0 & 12.0 & 18.0 & 30 & 67.0 & 3.0 \\
\hline \multirow[t]{4}{*}{ (Trial Pit2) } & 1.5 & 4.0 & 9.0 & 14 & 54.0 & 32.0 \\
\hline & 2.0 & 5.0 & 10.0 & 15 & 68.0 & 17.0 \\
\hline & 2.5 & 5.0 & 12.0 & 17 & 43.0 & 40.0 \\
\hline & 3.0 & 7.0 & 35.0 & 42 & 36.0 & 22.0 \\
\hline Quartz Schist-derived Soils & 1.0 & 5.0 & 23.0 & 28 & 85.0 & 12.0 \\
\hline \multirow[t]{4}{*}{ (Trial Pit3) } & 1.5 & 2.0 & 20.0 & 22 & 68.0 & 10.0 \\
\hline & 2.0 & 5.0 & 28.0 & 33 & 59.0 & 8.0 \\
\hline & 2.5 & 6.0 & 18.0 & 24 & 54.2 & 22.0 \\
\hline & 3.0 & 3.0 & 37.0 & 40 & 38.0 & 22.0 \\
\hline Quartz Schist-derived Soils & 1.0 & 4.0 & 26.0 & 30 & 60.0 & 10.0 \\
\hline \multirow[t]{4}{*}{ (Trial Pit4) } & 1.5 & 7.0 & 24.0 & 31 & 37.0 & 32.0 \\
\hline & 2.0 & 7.0 & 24.0 & 31 & 51.0 & 18.0 \\
\hline & 2.5 & 7.0 & 19.0 & 26 & 56.0 & 18.0 \\
\hline & 3.0 & 5.0 & 15.0 & 20 & 58.0 & 21.0 \\
\hline Granite-derived Soils & 1.0 & 10.0 & 23.0 & 33 & 64.0 & 3.0 \\
\hline \multirow[t]{4}{*}{ (Trial Pit5) } & 1.5 & 5.0 & 39.0 & 45 & 54.0 & 1.0 \\
\hline & 2.0 & 8.0 & 39.0 & 47 & 51.0 & 2.0 \\
\hline & 2.5 & 5.0 & 37.0 & 42 & 28.0 & 30.0 \\
\hline & 3.0 & 2.0 & 14.0 & 16 & 51.0 & 33.0 \\
\hline Granite-derived Soils & 1.0 & 9.0 & 30.0 & 40 & 50.0 & 10.0 \\
\hline \multirow[t]{4}{*}{ (Trial Pit6) } & 1.5 & 6.0 & 22.0 & 28 & 61.0 & 11.0 \\
\hline & 2.0 & 9.0 & 69.0 & 78 & 15.0 & 7.0 \\
\hline & 2.5 & 8.0 & 21.0 & 29 & 65.0 & 2.0 \\
\hline & 3.0 & 8.0 & 23.0 & 31 & 68.0 & 1.0 \\
\hline
\end{tabular}


Table 3. Plasticity characteristics of the studied soils

\begin{tabular}{|c|c|c|c|c|c|c|c|c|c|c|c|c|c|}
\hline \multirow[t]{3}{*}{ Soil Type } & \multirow{3}{*}{$\begin{array}{l}\text { Depth } \\
(\mathrm{m})\end{array}$} & \multicolumn{4}{|c|}{ Consistency Limits } & \multicolumn{8}{|c|}{ Derived Limits } \\
\hline & & \multicolumn{2}{|c|}{$\begin{array}{l}\text { Liquid limit } \\
\qquad\left(\mathrm{L}_{\mathrm{L}}\right) \%\end{array}$} & \multicolumn{2}{|c|}{$\begin{array}{l}\text { Plastic limit } \\
\left(\mathrm{L}_{\mathrm{P}}\right) \% \\
\end{array}$} & \multicolumn{4}{|c|}{$\begin{array}{l}\text { Plasticity index } \\
\left(\mathrm{I}_{\mathrm{P}}\right) \%\end{array}$} & \multicolumn{2}{|c|}{$\begin{array}{c}\text { Consistency index } \\
\left(\mathrm{I}_{\mathrm{C}}\right) \\
\end{array}$} & \multicolumn{2}{|c|}{$\begin{array}{c}\text { Liquidity index } \\
\left(\mathrm{I}_{\mathrm{L}}\right) \\
\end{array}$} \\
\hline & & PIT 1 & PIT 2 & PIT 1 & PIT 2 & PIT 1 & $\mathrm{CV}$ & PIT 2 & $\mathrm{CV}$ & PIT 1 & PIT 2 & PIT 1 & PIT 2 \\
\hline \multirow{5}{*}{$\begin{array}{l}\text { Gneiss-derived } \\
\text { soils }\end{array}$} & 1.0 & 36.6 & 29.8 & 12.0 & 15.0 & 24.6 & & 14.8 & & 1.4 & 1.8 & -0.4 & -0.76 \\
\hline & 1.5 & 36.0 & 41.3 & 18.7 & 20.6 & 16.0 & $28.79 \%$ & 20.7 & $42.53 \%$ & 1.5 & 1.6 & -0.53 & -0.56 \\
\hline & 2.0 & 42.1 & 67.6 & 27.0 & 46.8 & 15.1 & & 21.6 & & 2.3 & 2.6 & -1.33 & -1.57 \\
\hline & 2.5 & 37.3 & 31.7 & 26.0 & 20.0 & 11.3 & & 11.7 & & 3.1 & 2.4 & -2.09 & -0.83 \\
\hline & 3.0 & 39.5 & 41.0 & 21.0 & 25.0 & 18.0 & & 16.0 & & 1.9 & 1.4 & -0.94 & -0.36 \\
\hline \multirow{6}{*}{$\begin{array}{l}\text { Quartz } \\
\text { schist-derived } \\
\text { soils }\end{array}$} & & PIT 3 & PIT 4 & PIT 3 & PIT 4 & PIT 3 & & PIT 4 & & PIT 3 & PIT 4 & PIT 3 & PIT 4 \\
\hline & 1.0 & 32.0 & 35.2 & 9.0 & 16.0 & 23.0 & & 19.2 & & 0.7 & 1.0 & 0.3 & -0.03 \\
\hline & 1.5 & 28.8 & 53.7 & 17.0 & 18.4 & 11.8 & $27.32 \%$ & 35.3 & $33.27 \%$ & 1.1 & 1.0 & -0.08 & -0.01 \\
\hline & 2.0 & 32.0 & 57.6 & 18.1 & 43.2 & 13.9 & & 14.4 & & 1.3 & 2.4 & 0.29 & -1.44 \\
\hline & 2.5 & 42.6 & 39.1 & 11.0 & 13.0 & 21.6 & & 26.1 & & 0.8 & 0,9 & 0.19 & 0.15 \\
\hline & 3.0 & 44.7 & 60.3 & 25.0 & 30.6 & 19.7 & & 29.7 & & 1.3 & 2.0 & -0.25 & -0.75 \\
\hline \multirow{6}{*}{$\begin{array}{l}\text { Granite-derived } \\
\text { soils }\end{array}$} & & PIT 5 & PIT 6 & PIT 5 & PIT 6 & PIT 5 & & PIT 6 & & PIT 5 & PIT 6 & PIT 5 & PIT 6 \\
\hline & 1.0 & 28.0 & 48.9 & 18.8 & 19.5 & 9.2 & & 29.4 & & 2.9 & 1.1 & -1.89 & -0.08 \\
\hline & 1.5 & 29.9 & 34.5 & 17.0 & 29.3 & 12.9 & $39.38 \%$ & 5.2 & $75.73 \%$ & 2.2 & 4.3 & -1.15 & -3.26 \\
\hline & 2.0 & 45.0 & 35.3 & 38.0 & 23.7 & 7.0 & & 11.6 & & 6.0 & 2.0 & -5.0 & -0.95 \\
\hline & 2.5 & 41.2 & 38.4 & 35.5 & 13.6 & 5.7 & & 13.8 & & 6.0 & 0.1 & -5.0 & -0.06 \\
\hline & 3.0 & 28.0 & 47.4 & 13.0 & 14.0 & 15.0 & & 33.4 & & 1.5 & 1.1 & -0.47 & -0.12 \\
\hline
\end{tabular}

Table 4a. Consistency index (Ic) of the studied soils

\begin{tabular}{llll}
\hline Soil Type & Ic Range & State Of & Field Condition \\
\hline Gneiss-derived Soils & $1.4-3.1$ & Solid-hard & Unstable-Stable \\
Quartz schist-derived Soils & $0.8-2.4$ & Stiff-solid- hard & Unstable \\
Granite-derived soils & $0.1-6.0$ & Soft-solid-hard & Unstable-Stable \\
\hline
\end{tabular}

Table 4b. Liquidity index of the studied soils

\begin{tabular}{lll}
\hline Soil Type & $\mathrm{I}_{1}$ & Constitency \\
\hline Gneiss-derived Soils & $(-2.09)-(-0.63)$ & Semi plastic - solid \\
Quartz schist-derived soils & $(-0.95)-(0.3)$ & Semi plastic - plastic \\
Granite-derived soils & $(-5)-(-2.06)$ & Semi plastic - solid \\
\hline
\end{tabular}


Table 5. Major oxides geochemistry of lateritic soils (Adebisi, 2010)

\begin{tabular}{|c|c|c|c|c|c|c|c|c|c|c|c|c|c|c|c|}
\hline \multirow[b]{2}{*}{ Soil Type } & \multirow{2}{*}{$\begin{array}{l}\text { Depth } \\
\text { (m) }\end{array}$} & \multicolumn{14}{|c|}{ Amount Percent (\%) } \\
\hline & & $\mathrm{SiO}_{2}$ & $\mathrm{Al}_{2} \mathrm{O}_{3}$ & $\mathrm{TiO}_{2}$ & $\mathrm{Fe}_{2} \mathrm{O}_{3}$ & $\mathrm{CaO}$ & $\mathrm{MgO}$ & $\mathrm{K}_{2} \mathrm{O}$ & $\mathrm{Na}_{2} \mathrm{O}$ & $\mathrm{MnO}$ & $\mathrm{P}_{2} \mathrm{O}_{3}$ & $\mathrm{SO}_{3}$ & $\begin{array}{l}\mathrm{SiO}_{2} / \\
\mathrm{Fe}_{2} \mathrm{O}_{3}+\mathrm{Al}_{2} \mathrm{O}_{3}\end{array}$ & $\mathrm{CV}$ & $\begin{array}{l}\text { Soil } \\
\text { classification }\end{array}$ \\
\hline \multirow{2}{*}{$\begin{array}{l}\text { Gneiss-derived } \\
\text { soils }\end{array}$} & 1.0 & 39.50 & 29.16 & 1.10 & 17.67 & 0.07 & 0.33 & 1.29 & 0.10 & 0.03 & 0.05 & ND & 0.84 & & True laterite \\
\hline & 2.0 & 44.30 & 33.14 & 1.70 & 15.22 & 0.10 & 0.45 & 1.22 & 0.02 & 0.01 & 0.12 & ND & 0.91 & $5.7 \%$ & True laterite \\
\hline \multirow[t]{2}{*}{ PIT 1} & 3.0 & 43.60 & 30.45 & 1.04 & 16.88 & 0.02 & 0.35 & 1.02 & 0.06 & 0.03 & 0.06 & 0.04 & 0.94 & & True laterite \\
\hline & $\begin{array}{r}\text { Parent } \\
\text { Rock }\end{array}$ & 54.35 & 15.68 & 1.05 & 7.52 & 5.28 & 1.96 & 4.92 & 1.34 & 0.09 & 0.28 & ND & & & \\
\hline \multirow{2}{*}{$\begin{array}{l}\text { Gneiss-derived } \\
\text { soils }\end{array}$} & 1.0 & 41.30 & 31.12 & 3.00 & 15.87 & 0.15 & 0.53 & 1.27 & 0.05 & 0.02 & 0.10 & ND & 0.88 & & True laterite \\
\hline & 2.0 & 42.28 & 31.50 & 1.90 & 15.18 & 0.06 & 0.35 & 1.26 & 0.06 & 0.02 & 0.10 & ND & 0.91 & $3.3 \%$ & True laterite \\
\hline \multirow[t]{2}{*}{ PIT 2} & 3.0 & 43.54 & 32.45 & 1.00 & 13.69 & 0.08 & 0.47 & 1.01 & 0.04 & 0.02 & 0.09 & ND & 0.94 & & True laterite \\
\hline & $\begin{array}{r}\text { Parent } \\
\text { Rock }\end{array}$ & 56.32 & 13.70 & 1.17 & 9.50 & 5.14 & 1.96 & 3.92 & 2.32 & 0.12 & 0.24 & 0.02 & & & \\
\hline \multirow{4}{*}{$\begin{array}{l}\text { Quartz } \\
\text { schist-derived } \\
\text { soils } \\
\text { PIT } 3\end{array}$} & 1.0 & 57.52 & 21.18 & 1.18 & 6.70 & 0.80 & 1.80 & 3.54 & 0.40 & 0.21 & 0.10 & ND & 2.03 & & Non-laterite \\
\hline & 2.0 & 46.98 & 24.61 & 1.43 & 9.30 & 0.54 & 1.63 & 2.17 & 0.18 & 0.10 & 0.07 & ND & 1.39 & $25.5 \%$ & Lateritic soil \\
\hline & 3.0 & 47.66 & 27.46 & 1.15 & 9.20 & 0.30 & 1.43 & 2.32 & 0.17 & 0.18 & 0.06 & ND & 1.30 & & Lateritic soil \\
\hline & $\begin{array}{r}\text { Parent } \\
\text { Rock }\end{array}$ & 90.35 & 0.23 & 0.04 & 0.25 & 0.01 & 0.38 & 0.05 & 0.03 & 0.00 & 0.01 & ND & & & \\
\hline \multirow{4}{*}{$\begin{array}{l}\text { Quartz } \\
\text { schist-derived } \\
\text { soils } \\
\text { PIT } 4\end{array}$} & 1.0 & 56.50 & 19.18 & 1.38 & 7.69 & 0.88 & 1.94 & 2.64 & 0.35 & 0.27 & 0.05 & ND & 2.10 & & Non-laterite \\
\hline & 2.0 & 48.94 & 26.59 & 1.23 & 8.38 & 0.34 & 1.51 & 3.15 & 0.21 & 0.04 & 0.10 & ND & 1.40 & $26.1 \%$ & Lateritic soil \\
\hline & 3.0 & 47.70 & 25.50 & 1.25 & 10.14 & 0.42 & 1.53 & 1.47 & 0.14 & 0.24 & 0.04 & ND & 1.34 & & Lateritic soil \\
\hline & $\begin{array}{r}\text { Parent } \\
\text { Rock }\end{array}$ & 96.97 & 0.23 & 0.00 & 0.45 & 0.01 & 0.18 & 0.00 & 0.06 & 0.00 & 0.01 & ND & & & \\
\hline \multirow{2}{*}{$\begin{array}{l}\text { Granite-derived } \\
\text { soils }\end{array}$} & 1.0 & 42.44 & 27.76 & 1.25 & 16.20 & 0.08 & 0.40 & 0.32 & 0.03 & 0.03 & 0.14 & 0.02 & 0.96 & & True laterite \\
\hline & 2.0 & 43.75 & 27.03 & 1.96 & 19.25 & 0.08 & 0.40 & 0.80 & 0.02 & 0.03 & 0.13 & ND & 0.95 & $9.2 \%$ & True laterite \\
\hline \multirow[t]{2}{*}{ PIT 5} & 3.0 & 37.99 & 30.62 & 1.55 & 16.05 & 0.14 & 0.50 & 0.40 & 0.03 & 0.02 & 0.06 & ND & 0.81 & & True laterite \\
\hline & $\begin{array}{r}\text { Parent } \\
\text { Rock }\end{array}$ & 70.90 & 14.70 & 0.48 & 2.61 & 1.70 & 0.70 & 7.52 & 2.22 & 0.03 & 0.21 & ND & & & \\
\hline \multirow{2}{*}{$\begin{array}{l}\text { Granite-derived } \\
\text { soils }\end{array}$} & 1.0 & 44.40 & 29.70 & 1.45 & 15.50 & 0.04 & 0.44 & 0.44 & 0.02 & 0.01 & 0.17 & 0.02 & 0.98 & & True laterite \\
\hline & 2.0 & 41.81 & 25.07 & 1.86 & 19.37 & 0.18 & 0.34 & 0.75 & 0.03 & 0.05 & 0.06 & ND & 0.94 & $6.6 \%$ & True laterite \\
\hline \multirow[t]{2}{*}{ PIT 6} & 3.0 & 39.95 & 32.62 & 1.65 & 14.08 & 0.08 & 0.62 & 0.50 & 0.02 & 0.01 & 0.10 & ND & 0.86 & & True laterite \\
\hline & $\begin{array}{l}\text { Parent } \\
\text { Rock }\end{array}$ & 68.96 & 12.74 & 0.28 & 2.61 & 1.86 & 0.64 & 8.44 & 3.14 & 0.04 & 0.17 & ND & & & \\
\hline
\end{tabular}

Table 6. Clay mineralogical composition of the lateritic soils (Adebisi, 2010)

\begin{tabular}{|c|c|c|c|c|c|c|c|c|c|c|c|}
\hline \multirow[t]{2}{*}{ Soil Type } & \multirow[t]{2}{*}{$\begin{array}{l}\text { Depth } \\
\text { (m) }\end{array}$} & \multicolumn{4}{|c|}{$\begin{array}{l}\text { Amount Of Kaolinite } \\
(\%)\end{array}$} & \multicolumn{3}{|c|}{$\begin{array}{l}\text { Amount of Illite } \\
(\%)\end{array}$} & \multicolumn{3}{|c|}{$\begin{array}{l}\text { Amount of Quartz } \\
(\%)\end{array}$} \\
\hline & & PIT1 & PIT2 & Range & $\mathrm{CV}$ & PIT1 & PIT2 & Range & PIT1 & PIT2 & Range \\
\hline \multirow[t]{4}{*}{ Gneiss-derived soils } & 1.0 & 67.97 & 73.91 & \multirow[t]{3}{*}{$57.91-73.91$} & \multirow[t]{3}{*}{$11.56 \%$} & 3.93 & 8.07 & \multirow[t]{3}{*}{$3.93-17.61$} & 28.08 & 18.01 & \multirow[t]{3}{*}{$18.01-28.08$} \\
\hline & 2.0 & 61.38 & 58.95 & & & 8.05 & 5.66 & & 27.93 & 27.71 & \\
\hline & 3.0 & 57.91 & 64.43 & & & 17.61 & 7.22 & & 22.38 & 18.56 & \\
\hline & & PIT 3 & PIT4 & \multirow[t]{4}{*}{$54.33-71.17$} & & PIT3 & PIT4 & \multirow[t]{4}{*}{$1.61-12.96$} & PIT3 & PIT4 & \multirow[t]{4}{*}{$20.72-37.80$} \\
\hline \multirow{4}{*}{$\begin{array}{l}\text { Quartzschist-derived } \\
\text { soils }\end{array}$} & 1.0 & 58.02 & 62.03 & & \multirow[t]{3}{*}{$9.29 \%$} & 12.96 & 1.61 & & 29.01 & 36.36 & \\
\hline & 2.0 & 59.75 & 71.17 & & & 4.88 & 8.11 & & 35.37 & 20.72 & \\
\hline & 3.0 & 54.33 & 63.20 & & & 7.87 & 6.13 & & 37.80 & 30.67 & \\
\hline & & PIT 5 & PIT6 & \multirow[t]{4}{*}{$52.31-75.45$} & & PIT5 & PIT6 & \multirow[t]{4}{*}{$2.27-17.18$} & PIT5 & PIT6 & \multirow[t]{4}{*}{$12.16-29.88$} \\
\hline \multirow[t]{3}{*}{ Granite-derived soils } & 1.0 & 75.45 & 66.52 & & \multirow[t]{3}{*}{$11.92 \%$} & 3.45 & 2.27 & & 21.10 & 21.21 & \\
\hline & 2.0 & 52.31 & 70.14 & & & 17.81 & 2.70 & & 29.88 & 12.16 & \\
\hline & 3.0 & 71.16 & 70.80 & & & 5.61 & 7.83 & & 22.24 & 21.37 & \\
\hline
\end{tabular}


Table 7a. Consistency limits in relation to clay mineralogy of lateritic soils (Adebisi, 2010)

\begin{tabular}{|c|c|c|c|c|c|c|}
\hline Soil Type & $\begin{array}{l}\text { Liquid limit } \\
\left(\mathrm{L}_{\mathrm{L}}\right) \% \text { Range }\end{array}$ & $\begin{array}{l}\text { Inferred clay } \\
\text { mineral (s) }\end{array}$ & $\begin{array}{l}\text { Plastic Limit } \\
\left(L_{P}\right) \% \text { Range }\end{array}$ & $\begin{array}{l}\text { Inferred clay } \\
\text { mineral (s) }\end{array}$ & $\begin{array}{l}\text { Plasticity Index } \\
\left(\mathrm{I}_{\mathrm{P}}\right) \% \text { Range }\end{array}$ & $\begin{array}{l}\text { Inferred clay } \\
\text { mineral (s) }\end{array}$ \\
\hline Gneiss-derived soils & $29.8-42.1$ & Kaolinite & $12.0-46.8$ & Illite and Kaolinite & $11.3-24.6$ & No Montmorillonite \\
\hline $\begin{array}{l}\text { Quartz } \\
\text { schist-derived soils }\end{array}$ & $28.8-60.3$ & $\begin{array}{l}\text { Illite and } \\
\text { Kaolinite }\end{array}$ & $9.0-43.2$ & Illite and Kaolinite & $11.8-35.3$ & No Montmorillonite \\
\hline Granite-derived soils & $28.0-48,9$ & Kaolinite & $13.8-38$ & Kaolinite & $5.2-33.4$ & No Montmorillonite \\
\hline
\end{tabular}

Table 7b. Variation in undrained cohesion of lateritic soils with depth

\begin{tabular}{|c|c|c|c|c|c|c|c|}
\hline Parent Rocks & Depth $(\mathrm{m})$ & & & ined $\mathrm{Coh}$ & on $(\mathrm{Cu})$ & & \\
\hline \multirow{6}{*}{ Gneiss } & & PIT 1 & Range & $\mathrm{CV}$ & PIT 2 & Range & $\mathrm{CV}$ \\
\hline & 1.0 & 110 & \multirow{5}{*}{$55-110$} & \multirow{5}{*}{$38.52 \%$} & 25 & \multirow{5}{*}{$25-115$} & \multirow{5}{*}{$63.26 \%$} \\
\hline & 1.5 & 55 & & & 115 & & \\
\hline & 2.0 & 95 & & & 45 & & \\
\hline & 2.5 & 70 & & & 35 & & \\
\hline & 3.0 & 150 & & & 60 & & \\
\hline \multirow{6}{*}{$\begin{array}{l}\text { Quartz } \\
\text { Schist }\end{array}$} & & PIT 3 & \multirow{6}{*}{$60-130$} & \multirow{6}{*}{$30.30 \%$} & PIT 4 & \multirow{6}{*}{$60-105$} & \multirow{6}{*}{$41.33 \%$} \\
\hline & 1.0 & 60 & & & 95 & & \\
\hline & 1.5 & 75 & & & 105 & & \\
\hline & 2.0 & 130 & & & 60 & & \\
\hline & 2.5 & 90 & & & 65 & & \\
\hline & 3.0 & 80 & & & 160 & & \\
\hline \multirow{6}{*}{ Granite } & & PIT 5 & \multirow{6}{*}{$20-85$} & \multirow{6}{*}{$73.13 \%$} & PIT 6 & \multirow{6}{*}{$5-95$} & \multirow{6}{*}{$69.23 \%$} \\
\hline & 1.0 & 85 & & & 95 & & \\
\hline & 1.5 & 40 & & & 5 & & \\
\hline & 2.0 & 20 & & & 75 & & \\
\hline & 2.5 & 20 & & & 75 & & \\
\hline & 3.0 & 24 & & & 25 & & \\
\hline
\end{tabular}

Table 8 shows the compressibility behaviour due to clay content of lateritic soils. The amount of settlement of a lateritic soil can be estimated from the measured coefficient of volume compressibility. Applying the standard of Carter (1983), it becomes possible to express the compressibility characteristics of the soils as shown in Table 8 . Soils developed over parent rocks of similar mineralogical composition exhibit medium to high compressibility characteristics at pressure range of $100-200 \mathrm{kN} / \mathrm{m}^{2}$. All the soils exhibit low to medium compressibility at pressure ranges of $200-400 \mathrm{kN} / \mathrm{m}^{2}$ and $400-800 \mathrm{kN} / \mathrm{m}^{2}$.

In order to establish the influence of parent rock on the settlements that could be caused by foundation load, the statistical t-test on the coefficient of volume compressibility of the studied soils was conducted and the data are summarized in Table 9. Differential settlement is bound to occur if the corners of a structure are on the foundation soils of different parent rocks at the 0.5 significance level. This is possible at specified depth and pressure ranges. The T-test data on the coefficient of compressibility showing likelihood of differential settlement of laterised foundation soils are shown in Table 9. 
Table 8. Compressibility behavior due to clay content of lateritic soils (Adebisi, 2010)

\begin{tabular}{|c|c|c|c|c|c|c|c|c|c|c|c|c|c|}
\hline \multirow{4}{*}{$\begin{array}{l}\text { Soil } \\
\text { Type }\end{array}$} & \multirow{4}{*}{$\begin{array}{l}\text { Depth } \\
\text { (m) }\end{array}$} & \multicolumn{12}{|c|}{ Pressure Range $\left(\mathrm{kN} / \mathrm{m}^{2}\right)$} \\
\hline & & \multicolumn{4}{|c|}{$100-200$} & \multicolumn{4}{|c|}{$200-400$} & \multicolumn{4}{|c|}{$400-800$} \\
\hline & & \multicolumn{12}{|c|}{ Compressibilty $(\mathrm{Mv}) \mathrm{m}^{2} / \mathrm{MN}$} \\
\hline & & PITI & PIT2 & Range & Character & PIT1 & PIT2 & Range & Character & PIT 1 & PIT 2 & Range & Character \\
\hline \multirow{5}{*}{$\begin{array}{l}\text { Gneiss-derived } \\
\text { soils }\end{array}$} & 1.0 & 0.402 & 0.311 & \multirow{5}{*}{$0.110-0.402$} & \multirow{5}{*}{$\begin{array}{l}\text { Medium-High } \\
\text { Compressibility }\end{array}$} & 0.215 & 0.214 & \multirow{5}{*}{$0.089-0.255$} & \multirow{5}{*}{$\begin{array}{l}\text { Low-Medium } \\
\text { Compressibility }\end{array}$} & 0.108 & 0.114 & \multirow{5}{*}{$0.050-0.162$} & \multirow{5}{*}{$\begin{array}{l}\text { Low-Medium } \\
\text { Compressibility }\end{array}$} \\
\hline & 1.5 & 0.159 & 0.316 & & & 0.188 & 0.255 & & & 0.097 & 0.131 & & \\
\hline & 2.0 & 0.181 & 0.305 & & & 0.157 & 0.213 & & & 0.126 & 0.114 & & \\
\hline & 2.5 & 0.164 & 0.317 & & & 0.183 & 0.203 & & & 0,095 & 0.162 & & \\
\hline & 3.0 & 0.110 & 0.333 & & & 0.089 & 0.191 & & & 0.050 & 0.153 & & \\
\hline \multirow{6}{*}{$\begin{array}{c}\text { Quartz } \\
\text { schist-derived } \\
\text { soils }\end{array}$} & & PIT3 & PIT4 & \multirow{6}{*}{$0.062-0.525$} & \multirow{6}{*}{$\begin{array}{l}\text { Low-High } \\
\text { Compressibility }\end{array}$} & PIT3 & PIT4 & \multirow{6}{*}{$0.053-0.274$} & \multirow{6}{*}{$\begin{array}{l}\text { Low-Medium } \\
\text { Compressibility }\end{array}$} & PIT3 & PIT 4 & \multirow{6}{*}{$0.054-0.139$} & \multirow{6}{*}{$\begin{array}{l}\text { Low-Medium } \\
\text { Compressibility }\end{array}$} \\
\hline & 1.0 & 0.188 & 0.525 & & & 0.231 & 0.236 & & & 0.120 & 0.106 & & \\
\hline & 1.5 & 0.168 & 0.505 & & & 0.183 & 0.274 & & & 0.091 & 0.122 & & \\
\hline & 2.0 & 0.170 & 0.439 & & & 0.109 & 0.256 & & & 0.089 & 0.137 & & \\
\hline & 2.5 & 0.151 & 0.331 & & & 0.152 & 0.274 & & & 0.118 & 0.138 & & \\
\hline & 3.0 & 0.062 & 0.460 & & & 0.053 & 0.265 & & & 0.054 & 0.139 & & \\
\hline \multirow{6}{*}{$\begin{array}{c}\text { Granite-derived } \\
\text { soils }\end{array}$} & & PIT5 & PIT6 & \multirow{6}{*}{$0.130-0.524$} & \multirow{6}{*}{$\begin{array}{l}\text { Medium-High } \\
\text { Compressibility }\end{array}$} & PIT5 & PIT6 & \multirow{6}{*}{$0.090-0.250$} & \multirow{6}{*}{$\begin{array}{l}\text { Low-Medium } \\
\text { Compressibility }\end{array}$} & PIT 5 & PIT 6 & \multirow{6}{*}{$0.11-0.125$} & \multirow{6}{*}{$\begin{array}{l}\text { Low-Medium } \\
\text { Compressibility }\end{array}$} \\
\hline & 1.0 & 0.173 & 0.273 & & & 0.191 & 0.215 & & & 0.099 & 0.112 & & \\
\hline & 1.5 & 0.522 & 0.524 & & & 0.252 & 0.214 & & & 0.053 & 0.13 & & \\
\hline & 2.0 & 0.521 & 0.423 & & & 0.215 & 0.190 & & & 0.100 & 0.11 & & \\
\hline & 2.5 & 0.434 & 0.456 & & & 0.090 & 0.250 & & & 0.114 & 0.125 & & \\
\hline & 3.0 & 0.130 & 0.348 & & & 0.231 & 0.219 & & & 0.121 & 0.11 & & \\
\hline
\end{tabular}

Table 9. T-test on coefficient of compressibility showing likelihood of differential settlement of laterized foundation soils

\begin{tabular}{|c|c|c|c|c|c|c|c|c|c|c|}
\hline \multirow{3}{*}{$\begin{array}{l}\text { Depth } \\
\text { (m) }\end{array}$} & \multirow{3}{*}{ Parent Rocks } & \multicolumn{9}{|c|}{ Pressure Range } \\
\hline & & \multicolumn{3}{|c|}{ (a) $100-200 \mathrm{kN} / \mathrm{m}^{2}$} & \multicolumn{3}{|c|}{ (a) $200-400 \mathrm{kN} / \mathrm{m}^{2}$} & \multicolumn{3}{|c|}{ (a) $400-800 \mathrm{kN} / \mathrm{m}^{2}$} \\
\hline & & $\begin{array}{l}\text { Computed } \\
\text { T value }\end{array}$ & $\begin{array}{l}\text { Tin } \\
\text { table }\end{array}$ & Remark & $\begin{array}{l}\text { Computed } \\
\text { T value }\end{array}$ & $\begin{array}{l}\text { Tin } \\
\text { table }\end{array}$ & Remark & $\begin{array}{l}\text { Computed } \\
T \text { value }\end{array}$ & $\begin{array}{l}\text { Tin } \\
\text { table }\end{array}$ & Remark \\
\hline \multirow{3}{*}{1.0} & $\begin{array}{l}\text { Gneiss/Quatz } \\
\text {-Schist }\end{array}$ & 1.0 & 0.816 & Significant & 0.0996 & 0.816 & Not significant & 0.8743 & 0.816 & Significant \\
\hline & $\begin{array}{l}\text { Quartz-Schist/G } \\
\text { ranite }\end{array}$ & 0.462 & 0.816 & $\begin{array}{l}\text { Not } \\
\text { significant }\end{array}$ & 0.1922 & 0.816 & Not significant & 0.6771 & 0.816 & $\begin{array}{l}\text { Not } \\
\text { significant }\end{array}$ \\
\hline & Gneiss/Granite & 0.395 & 0.816 & $\begin{array}{l}\text { Not } \\
\text { significant }\end{array}$ & 0.5265 & 0.816 & Not significant & 0.3608 & 0.816 & $\begin{array}{l}\text { Not } \\
\text { significant }\end{array}$ \\
\hline \multirow{3}{*}{2.0} & $\begin{array}{l}\text { Gneiss/Quatz } \\
\text {-Schist }\end{array}$ & 0.552 & 0.816 & $\begin{array}{l}\text { Not } \\
\text { significant }\end{array}$ & 0.9650 & 0.816 & Significant & 0.8541 & 0.816 & Significant \\
\hline & $\begin{array}{l}\text { Quartz-Schist/G } \\
\text { ranite }\end{array}$ & 0.529 & 0.816 & $\begin{array}{l}\text { Not } \\
\text { significant }\end{array}$ & 0.8545 & 0.816 & Significant & 0.7463 & 0.816 & $\begin{array}{l}\text { Not } \\
\text { significant }\end{array}$ \\
\hline & Gneiss/Granite & 0.287 & 0.816 & $\begin{array}{l}\text { Not } \\
\text { significant }\end{array}$ & 0.7403 & 0.816 & Not significant & 0.4028 & 0.816 & $\begin{array}{l}\text { Not } \\
\text { significant }\end{array}$ \\
\hline \multirow{3}{*}{3.0} & $\begin{array}{l}\text { Gneiss/Quatz } \\
\text {-Schist }\end{array}$ & 0.730 & 0.816 & $\begin{array}{l}\text { Not } \\
\text { significant }\end{array}$ & 0.7882 & 0.816 & Not significant & 0.6771 & 0.816 & $\begin{array}{l}\text { Not } \\
\text { significant }\end{array}$ \\
\hline & $\begin{array}{l}\text { Quartz-Schist/G } \\
\text { ranite }\end{array}$ & 0.847 & 0.816 & Significant & 0.6609 & 0.816 & Not significant & 0.7601 & 0.816 & $\begin{array}{l}\text { Not } \\
\text { significant }\end{array}$ \\
\hline & Gneiss/Granite & 0.903 & 0.816 & Significant & 0.3671 & 0.816 & Not significant & 0.8467 & 0.816 & Significant \\
\hline
\end{tabular}

The means difference is significant at the 0.5 level.

\section{Discussion}

Quartz is the most abundant mineral in the studied parent rocks, and occurs as equidimensional hard grains. Micas, which include muscovite and biotite, are flaky minerals present in the granite. Muscovite is resistant to weathering, while biotite is easily broken-down. Clay results mainly from the breakdown of feldspar minerals because they are very flaky, and therefore, have large surface areas. Feldspars are major constituents of the parent rocks, although; the clayey lateritic soils also contain silt-sized particles.

\subsection{Soil Particle Size Distribution}

The grading curves for the studied soils cover several log cycles of the semi-log paper, showing that they contain a variety of particle sizes, and are therefore well-graded. In addition to the wide range of sizes in the lateritic soils as indicated by grading curves, the grading characteristics of the studied soils are further summarized in 
Table 2. It can be seen that sand and silt fractions dominate the composition of the studied soils, in comparison to gravel and clay fractions. It is important to note that silt and clay constitute the amount of fines present in such soils. On the basis of the Unified Soil Classification System (USCS), soils with amount of fines ranging between 0 and $5 \%$, the soils are generally ascribed to well-graded class, while soils with amount of fines in the range 5-15\% are said to be well-graded clayey sand or gravel. Soils with amount of fines range of $15-35 \%$ are described as very clayey. The amount of fines in gneiss-derived soils range between $11 \%$ and $42 \%$, in quartz schist-derived soils it varies between $20 \%$ and $40 \%$, while in granite-derived soils its range is from $16 \%$ to $78 \%$. Quartz schist-derived soils and granite-derived soils have amount of fines, which fall outside the standard stated above.

\subsection{Soil Classification}

The plasticity index $\left(\mathrm{I}_{\mathrm{P}}\right)$ versus liquid limit $\left(\mathrm{L}_{\mathrm{L}}\right)$ plots on the classification chart of Casagrande show that the fine soils plot partly below and above the A-line, while none goes beyond the U-line which is the limit for natural soils. It can be said therefore that gneiss-derived soils are clayey silt whose liquid limits are more than $40 \%$ but less than $70 \%$. Quartz-schist-derived soils reveal silty clay of low to high plasticity and granite-derived soils are clayey silt with plasticity less than $30 \%$ and close to $50 \%$. Other derived limits of the soils are interpreted which include consistency index $\left(\mathrm{I}_{\mathrm{C}}\right)$ and liquidity index $\left(\mathrm{I}_{\mathrm{L}}\right)$. Consistency index can be employed to describe the susceptibility of some tropical soils to weathering and erosion (Lujan, 2003). From Table 4a, Ic of gneiss-derived soils range between 1.4 and 3.1. Quartz schist-derived soils have $\mathrm{I}_{\mathrm{C}}$ of between 0.8 and 2.4, while granite-derived soils have $\mathrm{I}_{\mathrm{C}}$ varying from 0.1 to 6.0. It follows that granite-derived soils are unstable except at depth of $2.0 \mathrm{~m}$ and $2.5 \mathrm{~m}$, while quartz schist-derived soils and gneiss-derived soils are generally stable.

Liquidity index $\left(\mathrm{I}_{\mathrm{L}}\right)$ of gneiss-derived soils range between -2.09 and -0.63 . $\mathrm{I}_{\mathrm{L}}$ for quartzite-derived soils varies from -0.95 to 0.3 , and $\mathrm{I}_{\mathrm{L}}$ for granite-derived soils range from -0.5 to -2.06 . Soils with $\mathrm{I}_{\mathrm{L}}$ greater than 1.0 are said to be liquid in state (Davison and Springman 2000). Except quartz schist-derived soils, which fall within the class of semi-plastic to solid, gneiss-derived soils and granite-derived soils whose parent rocks show similarity in mineralogical composition exhibit semi-solid to solid nature (Table 4b).

\subsection{Chemical Characterization}

The chemical data presented in Table 5, shows that the soils are enriched in the secondary oxides of iron (Fe) and aluminum (Al). Also they contain some easily soluble elements such as sodium ( $\mathrm{Na}$ ), potassium (K), calcium $(\mathrm{Ca})$, magnesium $(\mathrm{Mg})$ and silicon $(\mathrm{Si})$. Through this it is possible to compare the composition of fresh rock with that of soil developed over it, and distribution of the elements in order to explain weathering patterns (Bain et al, 1990). In this case, weathering resulted mainly in loss of $\mathrm{SiO}_{2}$ while there is enrichment of aluminum oxide $\left(\mathrm{Al}_{2} \mathrm{O}_{3}\right)$ and iron oxide $\left(\mathrm{Fe}_{2} \mathrm{O}_{3}\right)$ in the profiles. Classification of lateritic soils is also possible on the basis of the geochemistry of major oxides and in terms of silica-sesquioxide molar ratio. This was first introduced by Winterkorn et al. (1951) cited in Gidigasu (1976). Laterised soils derived from gneiss, quartz-schist and granite are thus classified as summarized in Table 5.

\subsection{Mineralogical Characterization}

Soils developed over quartz schist have less iron oxide than either of gneiss-derived soils or granite-derived soils. It must be born in mind that gneiss and granite have similar mineralogical composition. It is expected that the concentration of the major elements especially Fe, will either increase or decrease with depth depending on the season of the year. In a situation where it is not so, the coefficient of variation (CV) of these elements are determined with depth in order to explain their distribution in relation to laterisation.

Townsend et al. (1971) has shown that the sesquioxide content of engineering soils influences their geotechnical properties, especially, those employed in the design of road and highways. It follows that variation of subgrade material properties with depth can be defined. The Silica/sesquioxide molar ratio of iron and aluminum $\left(\mathrm{SiO}_{2} /\right.$ $\mathrm{Fe}_{2} \mathrm{O}_{3}+\mathrm{Al}_{2} \mathrm{O}_{3}$ ) shows variation of $26.1 \%$ with depth in the profiles over quartz schist, while silica-sesquioxide molar ratio shows a $\mathrm{CV}$ of $9.2 \%$ and a $\mathrm{CV}$ of $5.7 \%$ with depth in the profiles over gneiss and granite respectively. According to Rag (1972), variation below 10\% is indicative of good distribution. This is noted in soils developed over parent rocks, which are of different geological mode of formation but of similar mineralogical composition. It follows that laterisation is better distributed in the profiles developed over gneiss and granite than in the profiles developed over quartz-schist.

Quantitative interpretation of the X-Ray diffractograms shown in Table 6 reveals that kaolinite is the dominant clay mineral with subordinate amount of illite, while an insignificant amount (1.9 and $0.9 \%)$ of smectite, a clay mineral in the montmorillonite group occurred in the soils developed over rocks of similar mineralogical composition (granite and gneiss), at depth of $3.0 \mathrm{~m}$. This is not reflected in Table 6, but it is important to state it 
as smectite's presence is important due to its high swelling potential, which could make a soil unsuitable as subgrade or building foundation material. Kaolinite is known to be associated with a well-drained environment. In the profiles developed over rocks of similar mineralogical composition (gneiss and granite), the amount of kaolinite shows relatively greater variability than in the profile developed over quartz schist. It is also noted that quartz is the major non-clay mineral present in the studied soils. It is a known fact that geotechnical properties of lateritic soils are strongly influenced by the amount, and type of clay minerals present in it.

\subsection{Implications for Engineering Projects}

Mitchell (1993) shows that it is also possible to infer the various clay mineral types on the basis of the consistency limits of a lateritic soil. Soils derived from parent rocks with similar mineralogical composition (gneiss and granite) are clayey silt as against soils derived from quartz-schist. However, soils derived from parent rocks of similar (metamorphic) mode of formation show relatively lower variability in plasticity index with depth when compared to granite-derived soils. On the basis of liquid limit, kaolinite is present in soils developed over parent rocks of similar mineralogical composition, while the plastic limit reveals illite as the prominent clay minerals in soils developed from (Gneiss and quartz-schist) parent rocks of the same geological mode of formation. However, the plasticity index results reveal absence of montmorillonite which is known to constitute a nuisance when considering a lateritic soil as foundation material (Ola, 1981).

A very weak negative correlation $(r=-0.15)$ with a coefficient of determination $\left(\mathrm{r}^{2}\right)$ equal to 0.0229 can be noticed. The best line of fit for the relationship gives a regression equation $\mathrm{Ip}=-0.2686 \mathrm{Fe}_{2} \mathrm{O}_{3}+21.455$. The implication is that elevated amount of iron oxide reduces plasticity index of a lateritic soil. The scenario displayed in $7 \mathrm{~b}$ is the manner in which an engineering geologist handles the case. In this case, parent rock to each of the soils is given a serious consideration. Now, negative correlations exist between the plasticity index and amount of iron oxide in gneiss-derived soils $(r=-0.24)$ and granite-derived soils $(r=-0.79)$. This is due to the fact that gneiss-derived soils and granite-derived soils have higher amount of iron oxide than the quartz schist-derived soils. Igneous rock is known to be highly enriched in iron, strong inverse relationship between plasticity index and amount of iron oxide in granite-derived soils implies that the amount of iron oxide present in a granite-derived soil can be estimated if its plasticity index has been determined.

From Table 8, granite-derived soils tend to have less $\mathrm{Cu}$ values than gneiss-derived soils and quartz schist-derived soils. This may be due to the fact that both gneiss and quartz schist are of metamorphic origin as against granite, which is of igneous origin. In another development, the undrained cohesion of soils developed over gneiss and granite tend to have higher variation than soils developed over quartz schist. This situation can be explained in terms of similarities in mineralogical composition of the parent rocks.

Statistically, a hypothesis is tested at 0.05 to 0.1 levels of significance. The values of lateritic soils developed over two different parent rocks presented in Table 9 have significant difference at 0.5 level. This is indicative of differential settlement of small magnitude, which is geotechnically unacceptable as structures found on the soil stand a high chance of failure.

\section{Conclusions}

Compositional features of parent rocks and those of clay content of lateritic soils developed over them in parts of the Basement Complex terrain of Southwestern Nigeria were discussed. Properties which are peculiar to clay content have also been well analyzed. From the discussion of various results, the following conclusions can be drawn: A critical look at the thin sections reveals that gneiss and granite comprise nearly similar mineral composition despite differences in their mode of geological formation. Petrographic studies of the parent rocks revealed that quartz schist are composed of quartz and muscovite, while gneiss and granite comprise quartz, alkali feldspars and biotite. Quartz schist has less complex and fewer mineralogical constituents than gneiss and granite. Gneiss and quartz-schist are both metamorphic rocks, thus similar in their mode of geological formation. Gneiss and granite, though they differ in their geological mode of formation they still show similarity in their mineralogical composition.

Although, clay-sized particles are present, the relative proportions of the different grain sizes within the studied soil mass indicate that silt and sand fractions dominate the composition of the soils. The grain size distribution curves as well as values obtained for the coefficient of uniformity and the coefficient of curvature further corroborate the fact that the soils are well graded. Elemental abundances of the studied soils indicate that soils developed over gneiss and granite are characterized by greater amount of iron oxide than those developed over quartz schist. The determined silica-sesquioxide molar ratios show that laterisation in the horizon of soils developed over gneiss, decreases with depth while it increases with depth in the horizons of soils developed over quartz schist and granite. 
It is clear that lateritic soils developed over the Precambrian Basement Complex rocks are very clayey. Clay mineralogical composition of the studied soils indicate that kaolinite is the major clay minerals, with subordinate amount of illite, while quartz is the dominant non-clay mineral. However, insignificant amount of smectite occurs at depths $3.0 \mathrm{~m}$ in the profiles developed over gneiss and granite. Granite-derived soils tend to have less cohesive strength than gneiss-derived soils and quartz schist-derived soils due to the fact that both gneiss and quartz schist are of metamorphic origin as against granite, while soils developed over gneiss and granite tend to have higher variation than soils developed over quartz schist due to similarity in mineralogical composition of the parent rocks.

Regression plots between plasticity index and amount of iron oxide in the clay content of lateritic soils due to parent rock factor confirm that it is the form of existence of iron oxide, and not the total iron content that reduces the plasticity of lateritic soils. Coefficient of compressibility of the gneiss- and granite-derived soils shows greatest variabilities, which decrease with pressure range. This implies that building load will cause the amount of settlement to decrease with depth. Differential settlement of structure is likely to occur if its corners are founded on soils developed from two different rock types at depths $1.0 \mathrm{~m}, 2.0 \mathrm{~m} 3.0 \mathrm{~m}$, but this is subject to pressure.

\section{Acknowledgements}

Research support from the two universities: The Olabisi Onabanjo University and Wlter Sisulu University are warmly acknowledged.

\section{References}

Adebisi, N. O. (1999). Geological control of Eleyele dam: A case study of Ibadan metropolis. PGD RS/GIS project RECTAS O.A.U. Ile-Ife.

Adebisi, N. O. (2005). GIS technique applied to surface water survey in Southwestern Nigeria: A case study of Eleyele dam, Ibadan. Global Journal of Geological Sciences, 3(1), 35-38.

Adebisi, N. O. (2010). Geotechnical investigation of foundation soils in parts of Basement Complex terrain of Southwestern Nigeria (Doctoral thesis, Dept. of Geology, University of Ibadan, Nigeria).

Adeyemi, G. O. (1992). Highway geotechnical properties of laterised residual soils in the Ajebo-Ishara geological transition zone of southwestern Nigeria (Unpublished doctoral thesis, O.A.U., Ile-Ife, Nigeria).

Adeyemi, G. O. (1994). Clay mineralogy, major element geochemistry and strength characteristics of three highway subgrade soils in southwestern Nigeria. Bulletin of Engineering Geology and Environment, 50(1), 5-8. http://dx.doi.org/10.1007/BF02594951

Aleva, G. J. J. (1994). Laterites: Concepts, geology morphology and chemistry (p. 169). Wegeningen, The Northerlands: ISRC.

Bain, D. C., Mellor, A. Y., \& Wilson, M. J. (1990). Nature and origin of an aluminous vermiculitic weathering product in acid soils from upland catchments in Scotland. Clay Minerals, 25, 467-475. http://dx.doi.org/10.1180/claymin.1990.025.4.05

British, S. B. S. (1990). Methods of testing of soils for civil engineering purposes. British Standard Institution.

Carter, M. (1983). Geotechnical engineering handbook (p. 226). London: Pentech. Press.

Casagrande, A. (1932). Research on the Atterberg Limits of Soils. Public Roads, 12(3), 121-130, 136.

Chandrakaran, S., \& Nambiar, M. R. M. (1993). Role of Iron oxide on Shear strength behaviour of lateritic soils. Indian Geotechnical Journal, 24, 194-206.

Das, B. M. (2008). Advanced Soil Mechanics (p. 20). New York: Taylor \& Francis.

Davison, L., \& Springman S. (2000). Soil description and classification. University of West England. Retrieved September 13, 2002, from http://www.fbe.uwe.ac.uk/public/geocal/soilmech/classification/solclass.ntm

Donker, N. H. W. (1974). A selection of procedures for soil analysis and testing. ITC - International Report.

Elueze, A. A., Ekengele, N. I., \& Bolarinwa, A. T. (2004). Industrial assessment of the residual clay bodies over gneisses and schists of Youndé area, Southern Cameroon. Journal of Mining and Geololgy, 40(1), 9-15. http://dx.doi.org/10.4314/jmg.v40i1.18804

Faniran, A., \& Jeje, L. K. (1983). Humid tropical geomorphologic processes and landform in warm humid climates. Longman Edition. Retrieved June 10, 2007, from http://www.getcited.org/pub/102217815 
Gidigasu, M. D. (1976). Laterite soil engineering. Development in geotechnical engineering. Elsevier Scientific Publishing Company.

Ige, O. A., Durotoye, B., \& Oluyemi, A. E. (2005). Mineralogy and geochemistry of lateritic weathering profile on ultramafic rock bodies around Mokuro, Ile-Ife area, Southwestern Nigeria. Journal of Mining and Geololgy, 41(1), 11-18.

Jones, H. A., \& Hockey, R. D. (1964). The geology of parts of Southwestern Nigeria. Explanation of 1:250, 000, Sheet Nos 59 and 68. Geological Survev of Nigeria Publication, 11, 101.

Lujan, D. L. (2003). Soil physical properties affecting soil erosion in tropical soils. Facultad de Agronomia, Instituto de Edafologia, Universidad, Maracay, Venezuela. Lecture given at the College on Soil Physics, Trieste. pp. 234.

Mitchell, J. K. (1993). Fundamentals of soil behavior (2nd ed.). New York: John Wiley.

Oke, T. R. (1974). Review of urban climatology 1968-1973. WM. Technical Note no. 134, World Meteorological Organisation, Geneva.

Okonkwo, C. T. (2005). Geochemistry of quartzites and quartz-mica schist in Jebba area, Nigeria. Journal of Mining and Geololgy, 28, 203-209. http://dx.doi.org/10.4314/jmg.v40i2.18814

Ola, S. A. (1981). Mineralogical properties of some Nigerian residual soils in relation with building problems. Bulletin of Egineering geology, 15, 1-13.

Philpotts, A. R. (1989). Petrography of igneous and metamorphic rocks. Retrieved December 4, 2002, from http://www.geolab.Unc.edu/petunia/IgmetAtlas/meta-micro/metamicro.html

Rag, D. (1972). The design of sample surveys. New Delhi, India: McGraw Hill, L.T.D.

Rahaman, M. A. (1976). Review of the Basement geology of Southwestern Nigeria (pp. 41-58).

Rahaman, M. A. (1988). Recent advances in the study of the Basement Complex of Nigeria. Publication of Geological Survey of Nigeria, 1-44.

Shelley, D. (1993). Igneous and metamorphic rocks under the microscope: Classification, textures, microstructures and mineral preferred orientations (p. 445). New York: Chapman \& Hall.

Townsend, F. C. (1985). Geotechnical characteristics of residual soils. Geotechnical Engineering. Proceeding of American Society of Civil Engineers, 111, 77-93.

Townsend, F. C., Manke, G. P., \& Parcher, J. V. (1971). Influence of sesquioxides on lateritic soils properties. Highway Resources Bard and Reclamation, 374, 80-92.

Wilson, M. J. (1986). Mineral weathering processes in podzolic soils on granitic materials and their implications for surface water acidification. Journal of the Geological Society of London, 143, 691-697. http://dx.doi.org/10.1144/gsjgs.143.4.0691 